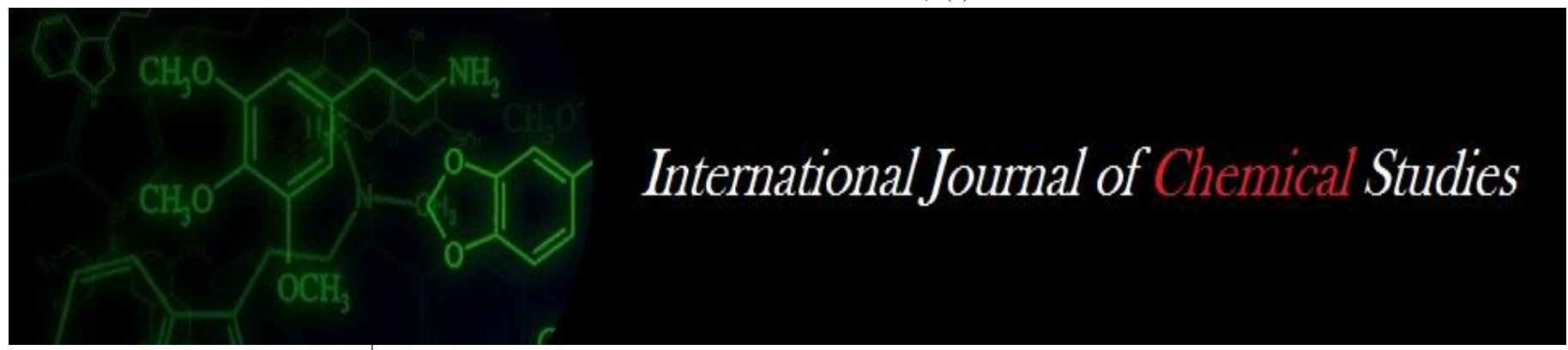

P-ISSN: 2349-8528

E-ISSN: 2321-4902

www.chemijournal.com

IJCS 2020; 8(2): 732-746

(C) 2020 IJCS

Received: 01-01-2020

Accepted: 05-02-2020

Parijat Bhattacharya

Ph.D. Research Scholar,

Department of Agricultural

Chemistry and Soil Science,

Bidhan Chandra Krishi

Viswavidyalaya, Mohanpur,

Nadia, West Bengal, India

Sudip Sengupta

Ph.D. Research Scholar,

Department of Agricultural

Chemistry and Soil Science,

Bidhan Chandra Krishi

Viswavidyalaya, Mohanpur,

Nadia, West Bengal, India

Sanjay Halder

Ph.D. Research Scholar, Department of Agricultural Chemistry and Soil Science, Bidhan Chandra Krishi

Viswavidyalaya, Mohanpur, Nadia, West Bengal, India

Corresponding Author: Sudip Sengupta

Ph.D. Research Scholar,

Department of Agricultural

Chemistry and Soil Science,

Bidhan Chandra Krishi

Viswavidyalaya, Mohanpur,

Nadia, West Bengal, India

\section{Characterization and delineation of micronutrient pools in some selected Inceptisols and Alfisols of West Bengal}

\author{
Parijat Bhattacharya, Sudip Sengupta and Sanjay Halder
}

DOI: $\underline{\text { https://doi.org/10.22271/chemi.2020.v8.i21.8856 }}$

\begin{abstract}
The present study has been conducted to determine the cationic micronutrient $(\mathrm{Zn}, \mathrm{Cu}, \mathrm{Fe}, \mathrm{Mn})$ pools based on other factors towards plant availability in 20 selected soil series (10 series each from Alfisols and Inceptisols) of West Bengal. Apart from physicochemical characterization, the soils were sequentially extracted for different fractions such as the water-soluble and exchangeable (SE); organically bound $(\mathrm{OB})$; manganese oxide-bound / occluded ( $\mathrm{MnOx})$, amorphous iron oxide-bound $\left(\mathrm{Am} \_\mathrm{FeOx}\right)$, crystalline oxide-bound (Cry_FeOx) fractions using sequential extraction process.

The percent predominance of the manganese fractions in the selected Alfisols and Inceptisols followed the order as $\mathrm{MnOx}>\mathrm{Am} \_\mathrm{FeOx}>\mathrm{Cry} \_\mathrm{FeOx}>\mathrm{OB}>\mathrm{SE}$ fractions while for copper, it followed the order as $\mathrm{OB}>\mathrm{Cry} \_\mathrm{FeOx}>\mathrm{Am} \_\mathrm{FeOx}>\mathrm{SE}>\mathrm{MnOx}$ fractions. The percent predominance of the iron fractions in the selected Alfisols and Inceptisols followed the order as Cry_FeOx $>\mathrm{Am} \_\mathrm{FeOx}>\mathrm{MnOx}>\mathrm{OB}>\mathrm{SE}$ fractions while for zinc, it followed the order as Am_FeOx $>$ Cry_FeOx $>\mathrm{MnOx}>\mathrm{OB}>\mathrm{SE}$ fractions. The different fractions of $\mathrm{Zn}, \mathrm{Cu}, \mathrm{Fe}, \mathrm{Mn}$ were then stepwise regressed with other fractions and soil physicochemical properties. The most phyto-available fraction i.e. soluble and exchangeable $\mathrm{Mn}, \mathrm{Cu}, \mathrm{Fe}$ and $\mathrm{Zn}$ was found to be mostly governed by clay content of soil and organically bound fractions of respective elements.
\end{abstract}

Keywords: Micronutrients, fractions, inceptisols, alfisols, regression

\section{Introduction}

Micronutrients are essential elements taken up by plants in minute quantities include iron $(\mathrm{Fe})$, manganese $(\mathrm{Mn})$, zinc $(\mathrm{Zn})$, copper $(\mathrm{Cu})$, molybdenum $(\mathrm{Mo})$, nickel $(\mathrm{Ni})$ boron $(\mathrm{B})$ and chlorine $(\mathrm{Cl})$. Out of the cationic micronutrients, iron $(\mathrm{Fe})$, manganese $(\mathrm{Mn})$, zinc $(\mathrm{Zn})$ and copper $(\mathrm{Cu})$ are the most important ones. Micronutrients can only exhibit their function when present in balanced proportion (Tavakoli et al., 2014) ${ }^{[70]}$.

Among these four elements, Fe and $\mathrm{Mn}$ are termed as redox element for having more than one stable oxidation state and therefore play important roles on oxidation and reduction processes, as electron transport in photosynthesis, respiration and other biochemical and physiological pathways (Millaleo et al., 2010; Mousavi et al., 2011) ${ }^{[21,45]}$. Manganese has important role on activation of several enzymes involved in oxidation reactions, carboxylation, carbohydrates metabolism, phosphorus reactions and citric acid cycle. It plays an important role in chlorophyll production and also involved in cell division and plant growth (Marschner, 2011; Tavakoli et al., 2014) ${ }^{[37,70]}$. The importance of iron is an lies in its essentiality for many important enzymes, including cytochrome that is involved in electron transport chain, chlorophyll synthesis etc. (Eskandari, 2011; Yadegari, 2014) ${ }^{[11,77]}$. The main functions of zinc lies in the tendency to make up tetragonal complexes with nitrogen, oxygen and sulfur, thus zinc have a catalytic, building and activating role in the enzymes (Mousavi et al., 2013; Maleki et al., $2014^{[45,34]}$. Zinc plays an important role in most of the enzymes like alcohol dehydrogenase carbonic anhydrase, superoxide dismutase, alkaline phosphatase, phosphatides lipase, carboxypeptidase, RNA polymerase, dehydrogenase and aldolase (Pandey et al., 2002; Mousavi et al., 2011) ${ }^{[52,45]}$. Copper is involved in several enzyme systems, cell wall formation and electron transport and oxidation reactions (ASK Saskatchewan Agriculture, 2012) ${ }^{[4]}$. Generally micronutrients in soils have been partitioned into different forms (McLaren and Crawford, 1973) ${ }^{[40]}$ or pools (Viets, 1962) ${ }^{[73]}$. 
These are soluble and exchangeable, organically bound, manganese oxide bound, amorphous iron oxide bound and crystalline iron oxide bound (Shuman, 1988; Shuman, 1991; Moreira et al., 2016) ${ }^{[61,58,44]}$.

Soluble and exchangeable (SE) pool although represents only a small fraction of the total metal content in soil, contributes greatly towards plant availability (Shukla \& Anshumali, 2018) ${ }^{[56]}$. Next to the SE fraction, micronutrients associated with soil organic matter via incorporation into organic molecules, exchange, chelation, or by specific and nonspecific adsorption have a considerable share in plant uptake (Shuman, 1991; Moreira et al., 2016) [61, 44]. Furthermore, micronutrient metals associated with iron and manganese oxides and hydrous oxides via adsorption, ion exchange, surface complex formations, incorporation into the crystal lattice and co-precipitation remain in a dynamic equilibrium with SE and OB fractions intimately affecting plant availability in soil system (Mandal and Mitra, 1982; Mandal and Mandal, 1987; Shuman, 1991) ${ }^{[36,35,61]}$.
A critical assessment of the different soil orders of West Bengal reveal that Alfisol and Inceptisol covers more than $75 \%$ of the area (Singh et al., 2009) ${ }^{[66]}$. The area are highly fertile and agriculturally productive (www.matirkotha.net) prioritizing the necessity to study of the soils from this area. On the above foreground, the study was catered with the objective of determining cationic micronutrient pools based on other factors towards plant availability.

\section{Materials and Methods \\ Site selection and sampling}

The experimental soils were collected from 20 different NBSS \& LUP identified soil series (10 soil series each from Inceptisol and Alfisol). The varying sites and their characteristics have been presented in Table-1.

\section{Physicochemical characterization}

The physico-chemical properties $(0-15 \mathrm{~cm})$ of the experimental soils from the 20 different soil series were analysed following standard methodologies as follows:-

\begin{tabular}{|c|c|c|}
\hline Parameters & Methodologies & References \\
\hline $\mathrm{pH}$ & Soil-water suspension $(1: 2.5)$ & Jackson, $1973^{[24]}$ \\
\hline EC & Soil-water suspension $(1: 2.5)$ & Jackson, $1967^{[23]}$ \\
\hline Clay content & Hydrometer method & Bouyoucos, $1962^{[7]}$ \\
\hline Organic Carbon & Wet oxidation method & Walkley and Black, $1934^{[74]}$ \\
\hline Available N & Hot alkaline permanganate & Subbiah and Asija, $1956^{[68]}$ \\
\hline \multirow{2}{*}{ Available P } & $0.5 \mathrm{M} \mathrm{NaHCO}_{3}(\mathrm{pH} 8.5)$ & Olsen and Sommers, $1982^{[50]}$ \\
\hline & $0.03 \mathrm{~N} \mathrm{NH}_{4} \mathrm{~F}+0.025 \mathrm{~N} \mathrm{HCl}(\mathrm{pH} 3.5)$ & Bray and Kurtz, $1945^{[8]}$ \\
\hline Available K & Neutral Normal ammonium acetate extraction & Knudsen, Peterson and Pratt, $1982^{[28]}$ \\
\hline Calcium Carbonate (\%) & Alkalimetric titration of acidified soil extract & Horváth et al., 2005 ${ }^{[21]}$ \\
\hline Available $\mathrm{Zn}, \mathrm{Mn}, \mathrm{Cu}, \mathrm{Fe}$ & DTPA extraction (pH 7.3) & Lindsay \& Norvell, $1978^{[31]}$ \\
\hline Amorphous $\mathrm{Fe}, \mathrm{Al}$ and $\mathrm{Mn}$ oxides & $0.2 \mathrm{M}$ acidified ammonium oxalate & McKeague and Day, $1966^{[39]}$ \\
\hline Total $\mathrm{Zn}, \mathrm{Mn}, \mathrm{Cu}$ and $\mathrm{Fe}$ & $\mathrm{HNO}_{3}: \mathrm{HClO}_{4}:: 10: 4(\mathrm{v} / \mathrm{v})$ digestion & Jackson, $1967^{[23]}$ \\
\hline
\end{tabular}

Determination of different pools of manganese, copper, iron \& zinc through fractionation of the experimental soils The soils have been extracted sequentially to determine different pools of manganese, copper, iron \& zinc by the standard methods as described Shuman (1985) ${ }^{[58]}$, Miller et al. (1986) ${ }^{[43]}$ and Phillips and Chappie (1995) ${ }^{[53]}$ modified by Golui et al. (2017) ${ }^{[17]}$. For execution, $2 \mathrm{~g}$ soil was treated sequentially with the series of extractants mentioned. After addition of each extractant to the soil, taken in a conical flask, the soil-extractant mixture was shaken in a reciprocating shaker, immediately centrifuged for 10 minutes at $2000 \mathrm{rpm}$ and the supernatant solution was decanted into another conical flask, while the remaining solid residue was subsequently treated for the next step of fractionation.

The different fractions thus obtained of cationic micronutrients $\mathrm{Zn}, \mathrm{Mn}, \mathrm{Cu} \& \mathrm{Fe}$ included water-soluble and exchangeable; organically bound; manganese oxide bound/occluded, amorphous iron oxide-bound and crystalline oxide-bound. Apart from this, the residual fractions i.e. carbonate bound and other was determined by subtracting the sum of the above-mentioned fractions from the total content.

\begin{tabular}{|c|c|c|c|c|}
\hline Steps & Fractions & Extractant & Soil: Solution & Conditions \\
\hline 1 & Water-soluble and exchangeable & $1 \mathrm{M} \mathrm{Ca}\left(\mathrm{NO}_{3}\right)_{2}{ }^{\mathrm{a}}$ & $1: 20$ & Shaking for 2 hours \\
\hline 2 & Organically bound & $\begin{array}{c}0.02 \mathrm{M} \mathrm{HNO}_{3}+30 \% \mathrm{H}_{2} \mathrm{O}_{2}(\mathrm{pH} 2.0)(\mathrm{vol} / \mathrm{vol})+3.2 \mathrm{M} \\
\mathrm{NH}_{4} \mathrm{OAc} \text { in } 20 \% \mathrm{HNO}_{3}(\mathrm{vol} / \mathrm{vol})^{\mathrm{c}}\end{array}$ & $1: 20$ & Shaking for 2.5 hours \\
\hline 3 & /occluded & $0.01 \mathrm{M} \mathrm{NH}_{2} \mathrm{OH} . \mathrm{HCl}+0.1 \mathrm{M} \mathrm{HNO}_{3}{ }^{\mathrm{b}}$ & $1: 20$ & Shaking fo \\
\hline 4 & Amorphous Fe ox & $0.2 \mathrm{M} \mathrm{NH}_{4}$-oxalate $+0.2 \mathrm{M} \mathrm{Oxalic}$ acid $^{\mathrm{a}}$ & $1: 20$ & Shaking for 4 hours in the dark \\
\hline 5 & Crystalline Fe oxide bound & $\begin{array}{c}0.2 \mathrm{M} \mathrm{NH}_{4-0 x a l a t e}+0.2 \mathrm{M} \mathrm{Oxalic} \mathrm{acid}+0.1 \mathrm{M} \\
\text { Ascorbic acid }\end{array}$ & $1: 20$ & $\begin{array}{l}\text { Boiling on water bath stir } \\
\text { occasionally for } 30 \text { minutes }\end{array}$ \\
\hline
\end{tabular}

a. Shuman (1985) ${ }^{[58]}$, b. Miller et al. (1986) ${ }^{[43]}$, c. Phillips and Chappie (1995) ${ }^{[53]}$ modified by Golui et al. (2017) ${ }^{[17]}$

Table 1: Selection criteria and location coordinates of the sites under study

\begin{tabular}{|c|c|c|c|c|c|c|}
\hline Block & Series & Taxonomic Unit & Textural class & Latitude & Longitude & Elevation \\
\hline \multicolumn{7}{|c|}{ Alfisol } \\
\hline Hura & Hura & Typic Haplustalfs & Sandy Clay Loam & $23.301^{\circ} \mathrm{N}$ & $86.659^{\circ} \mathrm{E}$ & $208 \mathrm{~m}$ AMSL \\
\hline Bundwan & Shimaldanga & Typic Haplustalfs & Loam & $22.876^{\circ} \mathrm{N}$ & $86.504^{\circ} \mathrm{E}$ & $219 \mathrm{~m}$ AMSL \\
\hline Puncha & Bishpuria & Ultic Haplustalfs & Sandy Loam & $23.158^{\circ} \mathrm{N}$ & $86.650^{\circ} \mathrm{E}$ & $180 \mathrm{~m}$ AMSL \\
\hline Manbazar & Sindurpur & Lithic Rhodustalfs & Sandy Loam & $23.398^{\circ} \mathrm{N}$ & $86.459^{\circ} \mathrm{E}$ & $249 \mathrm{~m}$ AMSL \\
\hline Puncha & Uparbaid & Typic Endoaqualfs & Sandy Clay Loam & $23.156^{\circ} \mathrm{N}$ & $86.649^{\circ} \mathrm{E}$ & $173 \mathrm{~m}$ AMSL \\
\hline
\end{tabular}




\begin{tabular}{|c|c|c|c|c|c|c|}
\hline Goaltor & Kusmasuli & Rhodic Paleustalfs & Sandy Loam & $22.697^{\circ} \mathrm{N}$ & $87.183^{\circ} \mathrm{E}$ & $65 \mathrm{~m}$ AMSL \\
\hline Goaltor & Barakadra & Aquic Haplustalfs & Loam & $22.702^{\circ} \mathrm{N}$ & $87.291^{\circ} \mathrm{E}$ & $44 \mathrm{~m}$ AMSL \\
\hline Jhargram & Jhargram & Ultic Paleustalfs & Loam & $22.452^{\circ} \mathrm{N}$ & $86.992^{\circ} \mathrm{E}$ & $84 \mathrm{~m}$ AMSL \\
\hline Teltaka & Teltaka & Aeric Endoaqualfs & Silt Loam & $22.753^{\circ} \mathrm{N}$ & $87.317^{\circ} \mathrm{E}$ & $52 \mathrm{~m}$ AMSL \\
\hline Kharagpur & Ruisanda & Vertic Endoaqualfs & Clay Loam & $22.354^{\circ} \mathrm{N}$ & $87.253^{\circ} \mathrm{E}$ & $43 \mathrm{~m}$ AMSL \\
\hline \multicolumn{7}{|c|}{ Inceptisol } \\
\hline Haringhata & Jaguli & Fluventic Eutrochrepts & Sandy Loam & $22.947^{\circ} \mathrm{N}$ & $88.539^{\circ} \mathrm{E}$ & $16 \mathrm{~m}$ AMSL \\
\hline Ranaghat & Ranaghat-I & Typic Ustrochrepts & Sandy Clay Loam & $23.167^{\circ} \mathrm{N}$ & $88.544^{\circ} \mathrm{E}$ & $12 \mathrm{~m}$ AMSL \\
\hline Ranaghat & Ranaghat-II & Aeric Haplaquepts & Clay & $23.204^{\circ} \mathrm{N}$ & $88.522^{\circ} \mathrm{E}$ & $13 \mathrm{~m}$ AMSL \\
\hline Tehatta & Tehatta & Aquic Ustifluvents & Clay Loam & $23.505^{\circ} \mathrm{N}$ & $88.555^{\circ} \mathrm{E}$ & $22 \mathrm{~m}$ AMSL \\
\hline Haringhata & Sirajanpara & Vertic Eutrochrepts & Clay Loam & $22.953^{\circ} \mathrm{N}$ & $88.525^{\circ} \mathrm{E}$ & $18 \mathrm{~m}$ AMSL \\
\hline Chakdaha & Maheshwarpur & Typic Haplustepts & Silty Clay Loam & $23.033^{\circ} \mathrm{N}$ & $88.549^{\circ} \mathrm{E}$ & $17 \mathrm{~m}$ AMSL \\
\hline Chakdaha & Pachpota & Aeric Haplustepts & Silty Loam & $23.074^{\circ} \mathrm{N}$ & $88.533^{\circ} \mathrm{E}$ & $16 \mathrm{~m}$ AMSL \\
\hline Chakdaha & Barabill & Typic Haplustepts & Clay & $23.078^{\circ} \mathrm{N}$ & $88.547^{\circ} \mathrm{E}$ & $16 \mathrm{~m}$ AMSL \\
\hline Haringhata & Laupala & Fluventic Eutrochrepts & Sandy Loam & $22.965^{\circ} \mathrm{N}$ & $88.547^{\circ} \mathrm{E}$ & $11 \mathrm{~m}$ AMSL \\
\hline Chakdaha & Kughachhi & Typic Haplustepts & Silty Clay & $23.046^{\circ} \mathrm{N}$ & $88.546^{\circ} \mathrm{E}$ & $12 \mathrm{~m}$ AMSL \\
\hline
\end{tabular}

\section{Statistical Interpretations}

The necessary computations of descriptive statistics of physicochemical properties and cationic micronutrient fractions and stepwise regression between those were performed using Microsoft Excel 2016 and SPSS version 23.0 (SPSS, Inc., Chicago, IL, USA).

\section{Results \\ Characterization of experimental soils}

The physico-chemical characteristics of these experimental soil series have been illustrated in table- 2, 3 and elucidated below.

The selected series of Alfisols (table-2) are slightly acidic in reaction $(\mathrm{pH} 6.140 \pm 0.501)$, non-saline in character $\left(\mathrm{EC}_{\mathrm{e}}\right.$ $\left.0.119 \pm 0.071 \mathrm{dSm}^{-1}\right)$, low in oxidizable organic carbon status $\left(4.913 \pm 1.298 \mathrm{~g} \mathrm{~kg}^{-1}\right)$ and low in clay fractions $(191.100 \pm$ $\left.83.901 \mathrm{~g} \mathrm{~kg}^{-1}\right)$. The selected soils are low in available (alkaline permanganate) nitrogen $\left(181.994 \pm 35.621 \mathrm{~kg} \mathrm{ha}^{-1}\right)$, moderate in available (Bray and Kutz No 1 reagent extractable) phosphorus (17.614 $\left.\pm 6.897 \mathrm{~kg} \mathrm{ha}^{-1}\right)$ and moderate in available $\left(\mathrm{NH}_{4} \mathrm{OAc}\right.$ extractable) potassium $\left(126.314 \pm 34.899 \mathrm{~kg} \mathrm{ha}^{-1}\right)$. The DTPA extractable micronutrients viz. $\mathrm{Zn}\left(1.209 \pm 0.239 \mathrm{mg} \mathrm{kg}^{-1}\right), \mathrm{Cu}(1.211 \pm$ $\left.0.339 \mathrm{mgkg}^{-1}\right), \mathrm{Fe}\left(44.426 \pm 12.056 \mathrm{mg} \mathrm{kg}^{-1}\right)$ and $\mathrm{Mn}(43.162$ $\left.\pm 11.699 \mathrm{mg} \mathrm{kg}^{-1}\right)$ are more or less sufficient. The recoveries of amorphous Fe $\left(7.132 \pm 0.621 \mathrm{~g} \mathrm{~kg}^{-1}\right)$ and Mn-oxide are relatively higher $\left(2.210 \pm 0.919 \mathrm{~g} \mathrm{~kg}^{-1}\right)$. The Calcium carbonate content was found to be $44.102 \pm 11.499 \mathrm{~g} \mathrm{~kg}^{-1}$. Therefore, ten selected soil series under Alfisols order under study varied significantly.

The selected series of Inceptisols (table-3) are neutral in reaction $(\mathrm{pH} 7.121 \pm 0.209)$, non-saline in character $\left(\mathrm{EC}_{\mathrm{e}}\right.$ $\left.0.289 \pm 0.081 \mathrm{dSm}^{-1}\right)$, moderate to high in oxidisable organic carbon status $\left(6.928 \pm 1.059 \mathrm{~g} \mathrm{~kg}^{-1}\right)$ and clay fractions $\left(346.210 \pm 119.899 \mathrm{~g} \mathrm{~kg}^{-1}\right)$. The selected soils are low to moderate in available (alkaline permanganate) nitrogen $\left(261.478 \pm 27.903 \mathrm{~kg} \mathrm{ha}^{-1}\right)$, high in available $\left(\mathrm{NaHCO}_{3}\right.$ extractable) phosphorus $\left(42.199 \pm 12.102 \mathrm{kgha}^{-1}\right)$ and moderate in available $\left(\mathrm{NH}_{4} \mathrm{OAc}\right.$ extractable) potassium $\left(225.891 \pm 32.995 \mathrm{~kg} \mathrm{ha}^{-1}\right)$. The DTPA extractable micronutrients viz. $\mathrm{Zn}\left(1.611 \pm 0.440 \mathrm{mg} \mathrm{kg}^{-1}\right), \mathrm{Cu}(1.279 \pm$ $\left.0.398 \mathrm{mgkg}^{-1}\right), \mathrm{Fe}\left(12.331 \pm 2.504 \mathrm{mg} \mathrm{kg}^{-1}\right)$ and $\mathrm{Mn}(11.609 \pm$ $\left.2.169 \mathrm{mg} \mathrm{kg}^{-1}\right)$ are more or less sufficient. The recoveries of amorphous Fe $\left(6.070 \pm 1.258 \mathrm{~g} \mathrm{~kg}^{-1}\right)$ was higher while that of Mn-oxide $\left(0.850 \pm 0.271 \mathrm{~g} \mathrm{~kg}^{-1}\right)$ are relatively lower. The Calcium carbonate content was found to be $50.798 \pm 13.392 \mathrm{~g}$ $\mathrm{kg}^{-1}$. A significant variation among the ten selected soil series under Inceptisols was observed.

\section{Recoveries of fractions of manganese, copper, iron \& zinc} of initial soils through sequential extraction

Following the standard protocols, the experimental soils were sequentially extracted for obtaining various pools of the micronutrients.

The water soluble and exchangeable (SE), organically bound (OB) manganese oxide-bound/occluded ( $\mathrm{MnOx}$ ), amorphous iron oxide-bound (Am_FeOx), crystalline iron oxide-bound (Cry_FeOx) and residual fractions of manganese, copper, iron \& zinc in Alfisols (Table-4) were observed to be in the ranges of 3.37-9.56 (5.94) mg kg-1, 16.88-34.13 (22.19) $\mathrm{mg} \mathrm{kg}$, 80.33-149.41 (113.55) $\mathrm{mg} \mathrm{kg}^{-1}, 32.35-77.85$ (52.58) $\mathrm{mg} \mathrm{kg}^{-1}$, 21.85-46.53 (36.01) $\mathrm{mg} \mathrm{kg}^{-1}$ and 37.21-90.4 (54.46) $\mathrm{mg} \mathrm{kg}^{-1}$; $0.315-1.273$ (0.80) $\mathrm{mg} \mathrm{kg}^{-1}, 7.535-14.204$ (10.36) $\mathrm{mg} \mathrm{kg}^{-1}$, $0.666-0.315(0.50) \mathrm{mg} \mathrm{kg}^{-1}, 5.365-9.208$ (6.71) $\mathrm{mg} \mathrm{kg}^{-1}$, 6.322-9.796 (7.51) $\mathrm{mg} \mathrm{kg}^{-1}$ and 9.678-14.057 (11.49) $\mathrm{mg} \mathrm{kg}^{-1}$; $159.48-487.63$ (364.27) $\mathrm{mg} \mathrm{kg}^{-1}, 850.57$ - 2030.1 (1451.14) $\mathrm{mg} \mathrm{kg}{ }^{-1}, 1709.37$ - 4255.68 (3050.26) $\mathrm{mg} \mathrm{kg}^{-1}, 2094.26-$ 4957.88 (3786) $\mathrm{mg} \mathrm{kg}^{-1}, 2466.65-5496.92$ (4075.85) $\mathrm{mg} \mathrm{kg}^{-1}$ and $2796.24-6279.98(4732.05) \mathrm{mg} \mathrm{kg}^{-1} ; 0.5-1.8(1.04) \mathrm{mg}$ $\mathrm{kg}^{-1}, 2.1-5.54$ (3.86) $\mathrm{mg} \mathrm{kg}^{-1}, 5.79-11.1$ (8.66) $\mathrm{mg} \mathrm{kg}^{-1}, 7.4-$ 18.71 (12.39) $\mathrm{mg} \mathrm{kg}^{-1}, 6.17-13.86$ (10.10) $\mathrm{mg} \mathrm{kg}^{-1}$ and 7.418.13 (14.27) $\mathrm{mg} \mathrm{kg}^{-1}$ respectively.

Similarly, the fractions of these cationic micronutrients i.e. water soluble and exchangeable (SE), organically bound (OB) manganese oxide-bound/occluded ( $\mathrm{MnOx}$ ), amorphous iron oxide-bound (Am_FeOx), crystalline iron oxide-bound (Cry_FeOx) and residual among the selected Inceptisols (Tabel-5) ranged from 4.34-12.38 (8.90) $\mathrm{mg} \mathrm{kg}^{-1}$, 39.44-65.67 (53.81) $\mathrm{mg} \mathrm{kg}^{-1}, 90.62-147.16$ (122.45) $\mathrm{mg} \mathrm{kg}^{-1}, 42.05-77.15$ (60.98) $\mathrm{mg} \mathrm{kg}^{-1}, 54.02-84.46$ (69.11) $\mathrm{mg} \mathrm{kg}^{-1}$ and 84.83146.24 (115.28) mg kg-1;0.46-1.78 (1.06) mg kg-1, 14.4823.23 (18.39) $\mathrm{mg} \mathrm{kg}^{-1}, 0.31-0.69$ (0.48) $\mathrm{mg} \mathrm{kg}^{-1}, 5.38-8.54$ (6.51) $\mathrm{mg} \mathrm{kg}^{-1}, 5.22-10.25$ (6.84) $\mathrm{mg} \mathrm{kg}^{-1}$ and $13.41-25.29$ (16.82) $\mathrm{mg} \mathrm{kg}^{-1}$; $306.02-1368.7$ (789.14) $\mathrm{mg} \mathrm{kg}^{-1}, 2986.56$ 6315.62 (4465.42) $\mathrm{mg} \mathrm{kg}^{-1}, 3742.44-6318.97$ (5010.94) $\mathrm{mg}$ $\mathrm{kg}^{-1}, 5295.33-9423.31(6830.45) \mathrm{mg} \mathrm{kg}{ }^{-1}, 7013.38-11077.4$ (8718.41) $\mathrm{mg} \mathrm{kg}^{-1}$ and 9282.42-16841.66 (11968.14) $\mathrm{mg} \mathrm{kg}^{-1}$; $0.64-2.9$ (1.53) mg kg-1, $4.46-11.93$ (8.78) $\mathrm{mg} \mathrm{kg}^{-1}, 5.27-13.56$ (9.42) $\mathrm{mg} \mathrm{kg}^{-1}, 11.35-27.12$ (19.01) $\mathrm{mg} \mathrm{kg}^{-1}, 5.27-16.27$ (9.90) $\mathrm{mg} \mathrm{kg}^{-1}$ and $13.46-38.61$ (23.37) $\mathrm{mg} \mathrm{kg}^{-1}$ respectively. The percent predominance of the manganese fractions in the selected Alfisols and Inceptisols followed the order as $\mathrm{MnOx}$ 
$>$ Am_FeOx > Cry_FeOx $>$ OB $>$ SE fractions while for copper, it followed the order as OB > Cry_FeOx $>$ Am_FeOx $>\mathrm{SE}>\mathrm{MnOx}$ fractions. The percent predominance of the iron fractions in the selected Alfisols and Inceptisols followed the order as $\mathrm{Cry} \_\mathrm{FeOx}>\mathrm{Am} \_\mathrm{FeOx}>\mathrm{MnOx}>\mathrm{OB}>\mathrm{SE}$ fractions while for zinc, it followed the order as Am_FeOx > Cry_FeOx $>\mathrm{MnOx}>\mathrm{OB}>\mathrm{SE}$ fractions. The mean values of different fractions of $\mathrm{Mn}, \mathrm{Cu}, \mathrm{Fe}$ and $\mathrm{Zn}$ as percentage of the total is represented as pie diagrams in the figures.

\section{Micronutrient fractions and soil physico-chemical properties: Relationship and Interrelationship}

The characterization of selected micronutrients $(\mathrm{Cu}, \mathrm{Mn}, \mathrm{Zn}$ and $\mathrm{Fe}$ ) in soil and the relative abundance of different fractions are largely governed by their relationship with soil physico-chemical properties and the interrelationship of the existing fractions as well. In the ensuing section, we tried to elucidate and understand such relationships and impacts thereof on soil micronutrients status.

Table 2: Physico-chemical parameters of selected Alfisols under study

\begin{tabular}{|c|c|c|c|c|c|c|c|c|c|c|c|c|c|c|}
\hline Soil series & pH & $\begin{array}{c}\mathbf{E C} \\
\left(\mathbf{d S S}^{-1}\right)\end{array}$ & $\begin{array}{c}\text { Clay } \\
\left(\mathrm{g} \mathrm{kg}^{-1}\right)\end{array}$ & $\begin{array}{l}\text { Org. C } \\
\left(\mathrm{g} \mathrm{kg}^{-1}\right)\end{array}$ & $\begin{array}{c}\mathbf{A v} \_\mathbf{N} \\
\left(\mathrm{kg} \mathrm{ha}^{-1}\right)\end{array}$ & $\begin{array}{c}\mathbf{A v} \_\mathbf{P} \\
\left(\mathbf{k g ~ h a}^{-1}\right)\end{array}$ & $\begin{array}{c}\text { Av_K } \\
\left(\mathbf{k g}_{\left.\mathbf{h} \mathbf{a}^{-1}\right)}\right.\end{array}$ & $\begin{array}{l}\mathrm{Am}-\mathrm{Fe} \\
\left(\mathrm{g} \mathrm{kg}^{-1}\right)\end{array}$ & $\begin{array}{l}\mathrm{CaCO}_{3} \\
\left(\mathrm{~g} \mathrm{~kg}^{-1}\right)\end{array}$ & $\begin{array}{l}\text { Mn-O } \\
\left(\mathrm{g} \mathrm{kg}^{-1}\right)\end{array}$ & 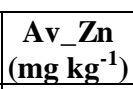 & $\begin{array}{c}\text { Av_Mn } \\
\left(\mathbf{m g} \mathbf{k g}^{-1}\right)\end{array}$ & 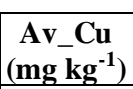 & 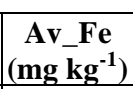 \\
\hline Hura & 5.910 & 0.210 & 364.000 & 5.700 & 148.600 & 12.544 & 112.200 & 6.250 & 44.300 & 0.940 & 1.131 & 30.050 & 1.546 & 30.450 \\
\hline Shimaldanga & 6.570 & 0.103 & 192.000 & 6.600 & 178.360 & 10.752 & 119.800 & 6.980 & 41.300 & 3.840 & 0.950 & 51.230 & 0.932 & 52.120 \\
\hline Bishpuria & 5.730 & 0.230 & 172.000 & 4.200 & 182.320 & 12.992 & 125.600 & 7.940 & 37.700 & 0.980 & 200 & 55.210 & 1.096 & 4.120 \\
\hline Sindurpur & 6.240 & 0.184 & 121.000 & 4.500 & 140.480 & 13.888 & 122.100 & 7.210 & 32.800 & 2.820 & 1.286 & 31.200 & 1.187 & 28.650 \\
\hline Uparbaid & 6.840 & 0.098 & 204.000 & 5.400 & 142.210 & 15.232 & 109.200 & 7.520 & 64.900 & 1.720 & 1.742 & 30.120 & 2.004 & 34.120 \\
\hline Kusmasuli & 6.750 & 0.074 & 168.000 & 3.600 & 182.670 & 30.016 & 222.800 & 6.510 & 44.900 & 3.210 & 63 & 230 & .874 & 5.020 \\
\hline Barakadra & 6.120 & 0.054 & 92.000 & 3.900 & 187.420 & 26.432 & 99.800 & 6.940 & 52.400 & \begin{tabular}{|l|}
2.010 \\
\end{tabular} & 183 & 60.100 & 1.030 & 53.560 \\
\hline Jhargram & 5.220 & 0.116 & 124.000 & 3.900 & 185.320 & 14.784 & 108.700 & 6.540 & 58.800 & 2.460 & 1.341 & 40.100 & 1.304 & 39.510 \\
\hline Teltaka & 6.080 & 0.069 & 162.000 & 4.200 & 214.880 & 25.536 & 126.400 & 8.160 & 32.700 & 1.850 & 1.370 & 32.240 & 0.963 & 55.370 \\
\hline Ruisanda & 5.870 & 0.057 & 302.000 & 7.500 & 258.340 & 13.888 & 116.700 & 7.240 & 31.100 & 2.250 & 0.968 & 53.110 & 1.124 & 40.250 \\
\hline Mean & 6.140 & 0.119 & 191.100 & 4.913 & 181.994 & 17.614 & 126.314 & 7.132 & 44.102 & 2.210 & 1.209 & 43.162 & 1.211 & 44.426 \\
\hline SD & 0.501 & 0.071 & 83.901 & 1.298 & 35.621 & 6.897 & 34.899 & 0.621 & 11.499 & \begin{tabular}{|l|}
0.919 \\
\end{tabular} & 0.239 & 11.699 & 0.339 & 12.056 \\
\hline
\end{tabular}

Table 3: Physico-chemical parameters of selected Inceptisols under study

\begin{tabular}{|c|c|c|c|c|c|c|c|c|c|c|c|c|c|c|}
\hline Soil series & pH & $\mathrm{EC}^{-1}$ & \begin{tabular}{|c|} 
Clay \\
$\left(\mathrm{g} \mathrm{kg}^{-1}\right)$
\end{tabular} & $\begin{array}{l}\text { Org. C } \\
\left(\mathrm{g} \mathrm{kg}^{-1}\right)\end{array}$ & $\begin{array}{l}\mathbf{A v} \_\mathbf{N} \\
\left(\mathrm{kg} \mathrm{ha}^{-1}\right)\end{array}$ & $\begin{array}{c}\mathbf{A v} \_\mathbf{P} \\
\left(\mathbf{k g ~ h a}^{-1}\right)\end{array}$ & $\begin{array}{c}\mathbf{A v} \_K \\
\left(\mathbf{k g ~ h a}^{-1}\right)\end{array}$ & A & 3 & -1 & $\begin{array}{c}\mathbf{A v} \_\mathbf{Z n} \\
\left(\mathbf{m g} \mathbf{k g}^{-1}\right)\end{array}$ & \begin{tabular}{|c} 
Av_Mn \\
$\left(\mathbf{m g} \mathbf{k g}^{-1}\right)$
\end{tabular} & 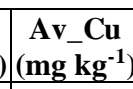 & 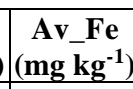 \\
\hline Jaguli & .120 & 0.245 & 144.000 & 6.600 & 265.400 & 37.632 & 229.800 & 4.560 & 42.800 & 0.660 & 2.486 & 12.300 & 1.406 & 10.450 \\
\hline & 240 & 0.202 & 340.000 & 8.400 & 286.480 & 60.032 & 294.600 & 8.460 & .700 & 1.540 & 1.660 & & 10 & 10 \\
\hline & 50 (1) & 82 & J2J. & 7.500 & & 2.256 & 0 & 6.420 & 00 & 990 & 960 & & & \\
\hline & 6.780 & 0.242 & 324. & 7.800 & 26 & 16 & 0 & 240 & 400 & 0.840 & 453 & & 973 & \\
\hline & 7.190 & 0.223 & & 6.3 & & & & & & & & & & \\
\hline Iahs & 6.870 & 0.372 & 364. & 7. & 0 & 4 & & 4. & 0 & 0 & 473 & & 682 & 10 \\
\hline & 7.55 & 12 & 279. & & & & & & & & & & & \\
\hline & 7.140 & 867 & 422.0 & 4. & 217 & 50.176 & 00 & 240 & 0 & 0.8 & .427 & & 100 & 50 \\
\hline & 020 & 0.258 & 182.000 & 7.500 & 292.340 & 25. & & 0 & 0 & 0.6 & 3 & & 902 & 40 \\
\hline & & & & & & & & & & & & & & \\
\hline $\mathrm{M}$ & 7.121 & 0.289 & 346.210 & \begin{tabular}{|l|l|}
6.928 \\
\end{tabular} & 261.478 & 42.199 & 225.891 & 6.070 & 50.798 & 0.850 & 1.6 & 11.609 & 279 & 12.331 \\
\hline $\mathrm{SD}$ & 0.209 & 0.081 & 119.899 & 1.059 & 27.903 & 12.102 & 32.995 & 1.258 & 13.392 & 0.271 & 0.440 & 2.169 & 0.398 & 2.504 \\
\hline
\end{tabular}

Table 4: Initial fractions of micronutrients for selected Alfisols $\left(\mathrm{mg} \mathrm{Kg}^{-1}\right)$

\begin{tabular}{|c|c|c|c|c|c|c|c|c|c|c|c|c|}
\hline \multicolumn{2}{|c|}{ Soil series } & Hura & Shimaldanga & Bishpuria & Sindurpur & Uparbaid & Kusmasuli & Barakadra & Jhargram & Teltaka & Ruisanda & Mean \\
\hline \multirow{7}{*}{ 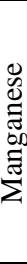 } & SE & 6.43 & 7.78 & 7.75 & 3.37 & 5.63 & 6.94 & 3.40 & 3.96 & 4.54 & 9.56 & 5.94 \\
\hline & OB & 17.14 & 31.13 & 26.42 & 17.55 & 17.31 & 19.55 & 24.59 & 17.16 & 16.88 & 34.13 & 22.19 \\
\hline & $\mathrm{MnOx}$ & 80.33 & 137.82 & 132.09 & 93.35 & 83.29 & 129.91 & 149.41 & 106.38 & 86.11 & 136.85 & 113.55 \\
\hline & Am_FeOx & 32.35 & 59.02 & 77.85 & 40.94 & 46.08 & 54.87 & & 43.82 & 44.14 & & 52.58 \\
\hline & Cry_FeOx & 33.20 & 41.51 & 38.40 & 28.34 & 26.83 & 43.83 & 46.53 & 38.27 & 21.85 & 41.29 & 36.01 \\
\hline & Residual & 44.77 & 47.02 & 69.75 & 41.39 & 37.21 & 60.23 & 90.40 & 54.38 & 42.84 & 56.65 & 54.46 \\
\hline & Total & 214.21 & 324.29 & 352.25 & 224.95 & 216.35 & 315.32 & 378.26 & 263.96 & 216.36 & 341.26 & 284.72 \\
\hline \multirow{7}{*}{$\begin{array}{l}\bar{\Delta} \\
\stackrel{0}{a} \\
\dot{0}\end{array}$} & SE & 1.27 & 080 & 077 & 054 & 127 & 0.66 & & 0.57 & 0.77 & 1.06 & 0.80 \\
\hline & OB & 12.25 & 10.00 & & 10.05 & 14.20 & 4 & & 5 & 95 & 11.36 & 10.36 \\
\hline & $\mathrm{MnOx}$ & 0.63 & 0.67 & 0.53 & 0.65 & 0.44 & 0.57 & 0.31 & 0.45 & 0.33 & 0.38 & 0.50 \\
\hline & Am_FeOx & 6.55 & 5.50 & 7.02 & 6.54 & 9.21 & 5.36 & 6.30 & 6.60 & 7.01 & 7.00 & 6.71 \\
\hline & Cry_FeOx & 9.29 & 6.66 & 6.32 & 7.23 & 9.80 & 6.33 & 7.00 & 8.31 & 6.64 & 7.57 & 7.51 \\
\hline & Residual & 12.25 & 9.70 & 10.99 & 11.13 & 14.06 & 9.68 & 11.89 & 12.56 & 12.17 & 10.49 & 11.49 \\
\hline & Total & 42.23 & 33.32 & 12 & 14 & 48.98 & 30.14 & & 38.14 & 36.87 & 37.86 & 37.38 \\
\hline \multirow{7}{*}{ :0ే } & SE & \begin{tabular}{|l|}
339.61 \\
\end{tabular} & & 422.73 & & & 487.63 & & 254.37 & 462.74 & 481.60 & 364.27 \\
\hline & OB & \begin{tabular}{|l|}
1018.83 \\
\end{tabular} & 2030.10 & 1517.99 & 850.57 & 1172.99 & 1551.55 & 1575.45 & 1271.85 & 1784.84 & 1737.20 & \begin{tabular}{|l|}
1451.14 \\
\end{tabular} \\
\hline & $\mathrm{MnOx}$ & \begin{tabular}{|l|}
1709.37 \\
\end{tabular} & 4060.20 & 2728.53 & 2009.47 & 2359.95 & 4255.68 & 3634.04 & 2984.61 & 3767.99 & 2992.80 & 3050.26 \\
\hline & Am_FeOx & 2094.26 & 3919.50 & 4419.45 & 2349.69 & 3183.84 & 4831.97 & 4621.32 & 3612.05 & 4957.88 & 3870.00 & 3786.00 \\
\hline & Cry_FeOx & 2886.68 & 172250 & 4131.23 & 2466.65 & 3281.59 & 5496.92 & 4810.37 & 4120.79 & 4781.60 & 4059.20 & 4075.85 \\
\hline & \begin{tabular}{|l|} 
Residual \\
\end{tabular} & 3271.57 & 0 & & 279 & 3602.76 & 5541.25 & & & 6279.98 & 4059.20 & 4732.05 \\
\hline & Total & 11320.30 & 20100.00 & 19215.00 & 10632.10 & 13964.20 & 22165.00 & 21006.00 & 16958.00 & 22035.00 & 17200.00 & 17459.56 \\
\hline \multirow{2}{*}{ 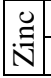 } & SE & 1.63 & 0.74 & 1.00 & 0.88 & 1.80 & 0.75 & 0.50 & 0.92 & 1.31 & 0.90 & 1.04 \\
\hline & OB & 4.35 & 2.96 & 3.39 & 4.56 & 5.54 & 2.10 & 3.59 & 4.00 & 4.86 & 3.22 & 3.86 \\
\hline
\end{tabular}




\begin{tabular}{|c|c|c|c|c|c|c|c|c|c|c|c|}
\hline MnOx & 8.15 & 6.17 & 6.79 & 11.10 & 11.09 & 6.44 & 9.38 & 11.07 & 10.60 & 5.79 & 8.66 \\
\hline Am_FeOx & 11.41 & 7.40 & 12.67 & 14.02 & 18.71 & 7.80 & 13.24 & 14.14 & 16.83 & 7.72 & 12.39 \\
\hline Cry_FeOx & 11.95 & 6.17 & 8.14 & 11.68 & 13.86 & 7.12 & 11.04 & 13.40 & 11.22 & 6.43 & 10.10 \\
\hline Residual & 16.84 & 7.40 & 13.26 & 16.18 & 18.30 & 9.70 & 17.44 & 17.96 & 17.52 & 8.11 & 14.27 \\
\hline Total & 54.33 & 30.83 & 45.24 & 58.41 & 69.31 & 33.92 & 55.18 & 61.49 & 62.34 & 32.17 & 50.32 \\
\hline
\end{tabular}

Table 5: Initial fractions of micronutrients for selected Inceptisols $\left(\mathrm{mg} \mathrm{Kg}^{-1}\right)$

\begin{tabular}{|c|c|c|c|c|c|c|c|c|c|c|c|c|}
\hline \multicolumn{2}{|c|}{ Soil series } & Jaguli & Ranaghat-I & Ranaghat-II & Tehatta & Sirajanpara & Maheshwarpur & Pachpota & Barabill & Laupala & Kughachhi & Mean \\
\hline \multirow{7}{*}{ 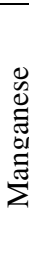 } & $\mathrm{SE}$ & 4.34 & 8.00 & 12.38 & 9.06 & 12.26 & 8.36 & 8.90 & 12.04 & 4.54 & 9.13 & 8.90 \\
\hline & OB & 54.54 & 61.62 & 48.81 & 65.67 & 57.74 & 50.97 & 55.89 & 48.15 & 55.30 & 39.44 & 53.81 \\
\hline & $\mathrm{MnOx}$ & 132.73 & 121.64 & 107.45 & 129.08 & 147.16 & 112.30 & 138.98 & 131.02 & 113.49 & 90.62 & 122.45 \\
\hline & Am_FeOx & 60.33 & 70.02 & 52.81 & 60.69 & 73.07 & 54.96 & 77.15 & 59.26 & 59.43 & 42.05 & 60.98 \\
\hline & Cry_FeOx & 84.46 & 54.02 & 55.36 & 76.09 & 78.18 & 67.30 & 75.67 & 80.09 & 63.55 & 56.39 & 69.11 \\
\hline & Residual & 146.24 & 84.83 & 87.42 & 112.32 & 142.56 & 104.34 & 137.99 & 132.41 & 116.38 & 88.34 & 115.28 \\
\hline & Total & 482.65 & 400.12 & 364.23 & 452.91 & 510.96 & 398.24 & 494.58 & 462.97 & 412.68 & 325.96 & 430.53 \\
\hline \multirow{7}{*}{$\begin{array}{l}\dot{\bar{d}} \\
\stackrel{\circ}{0} \\
\dot{o}\end{array}$} & SE & 0.46 & 1.06 & 1.47 & 0.85 & 0.96 & 1.28 & 0.96 & 1.78 & 0.46 & 1.28 & 1.06 \\
\hline & OB & 18.46 & 21.30 & 16.65 & 16.65 & 14.48 & 23.23 & 19.04 & 21.86 & 16.20 & 16.00 & 18.39 \\
\hline & $\mathrm{MnOx}$ & 0.47 & 0.69 & 0.48 & 0.42 & 0.31 & 0.56 & 0.45 & 0.60 & 0.38 & 0.42 & 0.48 \\
\hline & Am_FeOx & 6.08 & 7.99 & 5.62 & 5.38 & 5.42 & 7.03 & 7.50 & 8.54 & 5.79 & 5.73 & 6.51 \\
\hline & Cry_FeOx & 7.67 & 5.86 & 5.62 & 5.98 & 5.22 & 8.56 & 6.91 & 10.25 & 5.45 & 6.88 & 6.84 \\
\hline & Residual & 17.98 & 16.34 & 13.41 & 13.41 & 13.73 & 20.47 & 18.32 & 25.29 & 13.67 & 15.53 & 16.82 \\
\hline & Total & 51.12 & 53.24 & 43.25 & 42.69 & 40.12 & 61.12 & 53.19 & 68.32 & 41.96 & 45.84 & 50.09 \\
\hline \multirow{6}{*}{ 疋 } & $\mathrm{SE}$ & 306.02 & 780.12 & 1368.70 & 902.48 & 946.82 & 735.13 & 530.42 & 786.73 & 551.36 & 983.61 & 789.14 \\
\hline & $\mathrm{MnOx}$ & 4114.24 & 6318.97 & 5716.35 & 6227.11 & 5365.34 & 4655.80 & 3742.44 & 4084.97 & 5914.63 & 3969.58 & 5010.94 \\
\hline & Am_FeOx & 5746.34 & 7801.20 & 7246.08 & 7941.82 & 7298.44 & 5775.99 & 5628.39 & 5295.33 & 9423.31 & 6147.58 & 6830.45 \\
\hline & Cry_FeOx & 8330.49 & 7996.23 & 9057.60 & 10468.77 & 8758.12 & 8366.43 & 7013.38 & 7473.97 & 11077.40 & 8641.73 & 8718.41 \\
\hline & Residual & 11730.69 & 11038.70 & 11795.01 & 13943.32 & 12939.93 & 11236.93 & 9282.42 & 9631.44 & 16841.66 & 11241.28 & 11968.14 \\
\hline & Total & 34002.00 & 39006.00 & 40256.00 & 45124.00 & 39451.00 & 35006.00 & 29468.00 & 30259.00 & 50124.00 & 35129.00 & 37782.50 \\
\hline \multirow{7}{*}{ 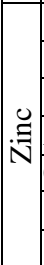 } & SE & 0.98 & 1.35 & 2.90 & 1.30 & 1.59 & 1.37 & 0.73 & 1.77 & 0.64 & 2.68 & 1.53 \\
\hline & OB & 11.93 & 10.09 & 11.07 & 9.07 & 7.29 & 8.17 & 4.46 & 6.81 & 7.59 & 11.31 & 8.78 \\
\hline & $\mathrm{MnOx}$ & 13.56 & 10.09 & 11.92 & 8.42 & 8.61 & 8.49 & 5.27 & 8.85 & 7.00 & 11.98 & 9.42 \\
\hline & Am_FeOx & 27.12 & 20.18 & 23.00 & 16.84 & 17.89 & 16.99 & 11.35 & 17.03 & 15.76 & 23.96 & 19.01 \\
\hline & Cry_FeOx & 16.27 & 7.40 & 11.07 & 9.07 & 8.61 & 9.15 & 5.27 & 10.22 & 7.59 & 14.38 & 9.90 \\
\hline & Residual & 38.61 & 18.16 & 25.21 & 20.08 & 22.26 & 21.17 & 13.46 & 23.43 & 19.79 & 31.53 & 23.37 \\
\hline & Total & 108.46 & 67.25 & 85.17 & 64.77 & 66.25 & 65.34 & 40.53 & 68.11 & 58.37 & 95.84 & 72.01 \\
\hline
\end{tabular}
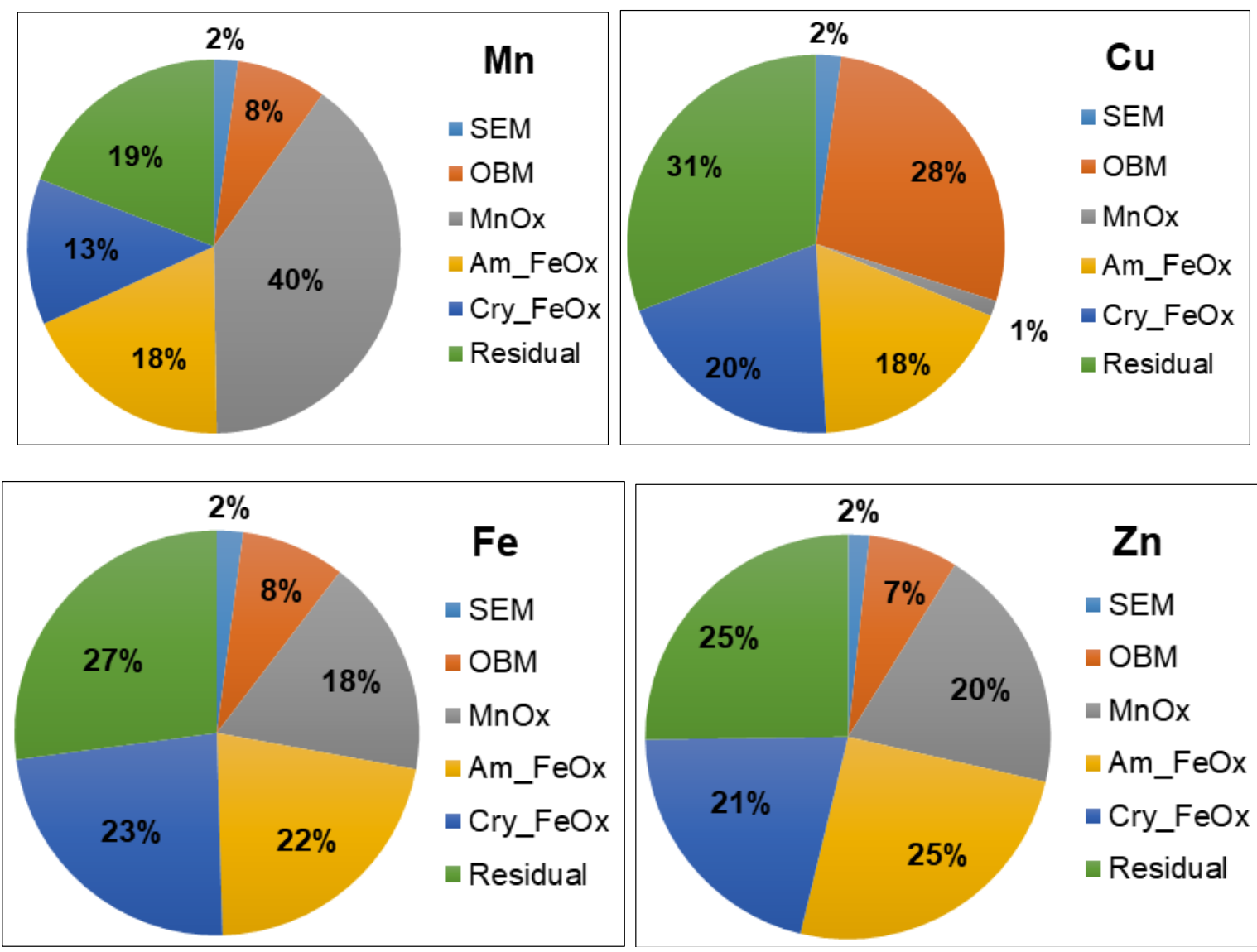

Fig 2: Initial fractions of manganese, copper, zinc and iron as $\%$ of the total in the selected Alfisols $\sim 736 \sim$ 

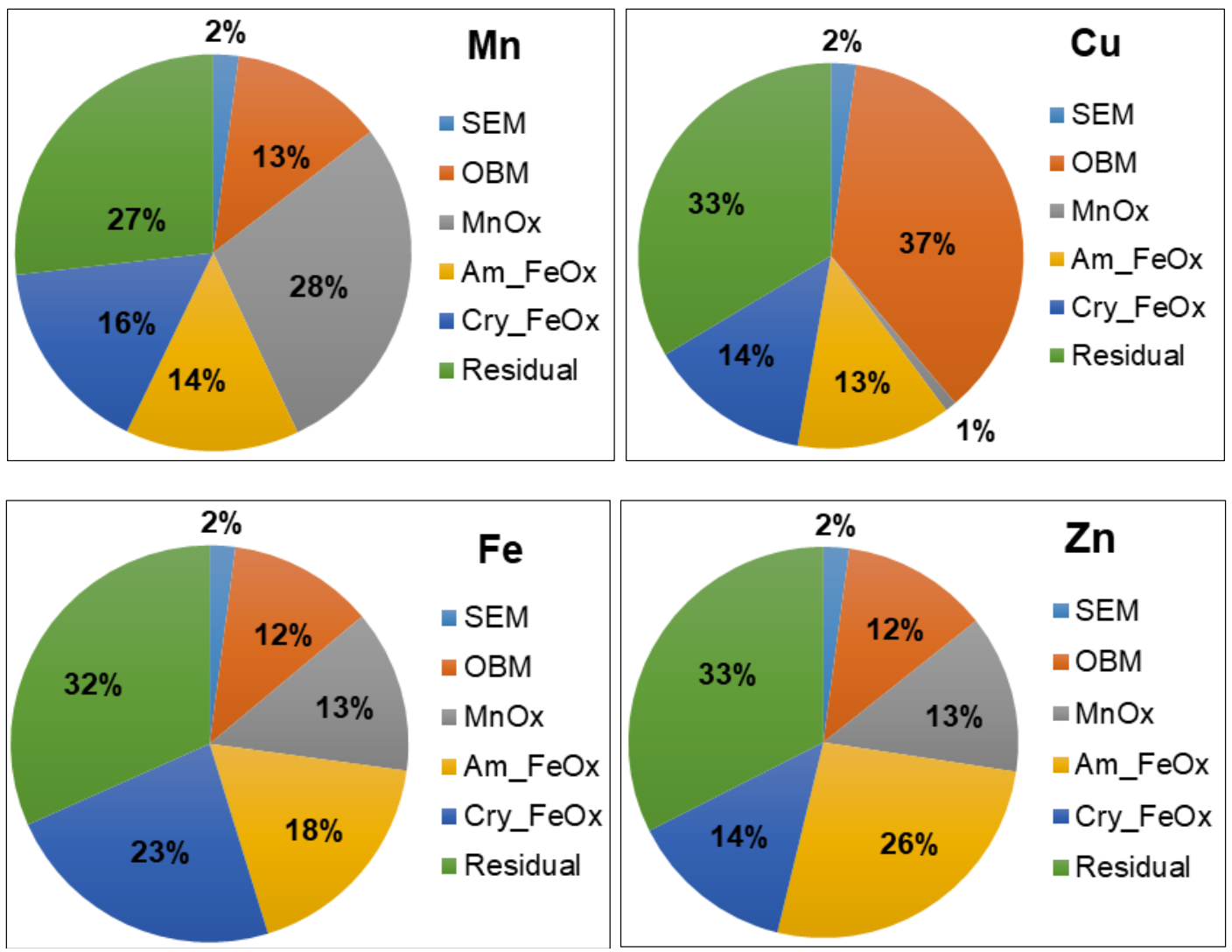

Fig 3: Initial fractions of manganese, copper, zinc and iron as $\%$ of the total in the selected Inceptisol

\section{Manganese}

Total and fractions of soil manganese were statistically regressed against soil physico-chemical properties. The inter relationship between $\mathrm{Mn}$ fractions have also been computed to assess the source-sink movement of soil Mn (Tables 6-7).

Table 6: Relationship and Interrelationship of Mn fractions in Alfisol

\begin{tabular}{|c|c|c|c|}
\hline & $\mathbf{R}^{2}$ & $\operatorname{Adj}-R^{2}$ & SEest. \\
\hline \multicolumn{4}{|c|}{ Between fractions } \\
\hline \multicolumn{4}{|l|}{ SE } \\
\hline $\mathrm{Y}=13.841+0.183 \mathrm{OB}^{*}$ & 0.863 & 0.744 & 0.255 \\
\hline \multicolumn{4}{|l|}{ OB } \\
\hline$Y=31.831-0.178$ Residual $*$ & 0.913 & 0.833 & 0.177 \\
\hline \multicolumn{4}{|l|}{$\mathrm{MnOx}$} \\
\hline $\mathrm{Y}=143.434+2.210$ Am_FeOx* & 0.915 & 0.837 & 0.956 \\
\hline \multicolumn{4}{|l|}{ Am_FeOx } \\
\hline $\mathrm{Y}=101.442+0.379 \mathrm{MnOx} *$ & 0.920 & 0.842 & 0.984 \\
\hline \multicolumn{4}{|l|}{ Cry_FeOx } \\
\hline $\mathrm{Y}=256.036-0.651$ Am_FeOx* & 0.921 & 0.842 & 0.968 \\
\hline \multicolumn{4}{|l|}{ Residual } \\
\hline $\mathrm{Y}=132.25-2.735 \mathrm{MnOx}^{* *}$ & 0.841 & 0.809 & 0.662 \\
\hline \multicolumn{4}{|c|}{ With physicochemical properties } \\
\hline \multicolumn{4}{|l|}{ SE } \\
\hline$Y=0.659+1.319$ Clay $*$ & 0.677 & 0.458 & 1.981 \\
\hline \multicolumn{4}{|l|}{ OB } \\
\hline $\mathrm{Y}=22.122+3.1270 \mathrm{OC}^{*}-1.567 \mathrm{Am} \_\mathrm{Fe}^{*}$ & 0.891 & 0.881 & 1.128 \\
\hline$Y=10.816+5.428 \mathrm{OC}^{*}$ & 0.780 & 0.760 & 1.516 \\
\hline \multicolumn{4}{|l|}{$\mathrm{MnOx}$} \\
\hline $\mathrm{Y}=10.969+2.224 \mathrm{MnO} *$ & 0.783 & 0.767 & 5.318 \\
\hline \multicolumn{4}{|l|}{ Am_FeOx } \\
\hline $\mathrm{Y}=54.660+8.891 \mathrm{Am} \_\mathrm{Fe}^{*}$ & 0.788 & 0.729 & 7.087 \\
\hline \multicolumn{4}{|l|}{ Cry_FeOx } \\
\hline $\mathrm{Y}=48.14-8.504 \mathrm{MnO}^{*}-5.514 \mathrm{Am} \_\mathrm{Fe}^{*}$ & 0.864 & 0.746 & 3.657 \\
\hline $\mathrm{Y}=68.701-5.507 \mathrm{MnO}^{* *}$ & 0.763 & 0.712 & 9.654 \\
\hline \multicolumn{4}{|l|}{ Residual } \\
\hline $\mathrm{Y}=83.139-9.642$ Am_Fe**_ $4.057 \mathrm{OC}^{*}$ & 0.952 & 0.907 & 8.443 \\
\hline $\mathrm{Y}=67.337-9.425 \mathrm{Am} \_\mathrm{Fe} *$ & 0.863 & 0.744 & 13.112 \\
\hline
\end{tabular}


The changes in soluble and exchangeable manganese (SE$\mathrm{Mn})$ are mostly governed by clay content of soil $(45.8 \%$ in Alfisols and $72.6 \%$ in Inceptisols) and organically bound manganese (74.4\% in Alfisols and $78.4 \%$ in Inceptisols). Organically bound manganese (OB-Mn) content is mostly governed by oxidizable organic carbon and amorphous iron oxide content in Alfisols (88.1\%) while in Inceptisols, oxidizable organic carbon largely controls the variation of OB-Mn (76.4\%) Among Mn fractions, residual manganese was the major contributory (dictates $83.3 \%$ variation of OB$\mathrm{Mn}$ in Alfisol and $81.2 \%$ in Inceptisol). Variations in MnOx$\mathrm{Mn}$ are mostly governed by manganese oxide content of soil (76.7\% in Alfisols) and manganese oxide and soil clay content (93.9\% in Inceptisols) and amorphous iron oxide bound $\mathrm{Mn}$ fraction (83.7\% in Alfisols and $85.1 \%$ in Inceptisols). Amorphous iron oxide bound fraction is largely impacted by changes in amorphous iron oxide content in Alfisols (72.9\%) and amorphous iron oxide content and soil clay content of Inceptisols $(80.4 \%)$. Manganese oxide bound Mn fractions was observed to dictate variations in amorphous iron oxide bound $\mathrm{Mn}$ (84.2\% in Alfisols and $82.5 \%$ in Inceptisols). Crystalline iron oxide bound fraction is mostly affected by variations in amorphous iron oxide and manganese oxide content in Alfisols (74.6\%) and by variations in manganese oxide in Inceptisols (60.3\%). Cry_FeOx-Mn was observed further to be largely governed by changes in amorphous iron oxide bound fraction $(84.2 \%$ in Alfisols and $85.2 \%$ in Inceptisols).

Table 7: Relationship and Interrelationship of Mn fractions in Inceptisols

\begin{tabular}{|c|c|c|c|}
\hline & $\mathbf{R}^{2}$ & Adj-R $\mathbf{R}^{2}$ & SEest. \\
\hline \multicolumn{4}{|l|}{ Between fractions } \\
\hline \multicolumn{4}{|l|}{ SE } \\
\hline $\mathrm{Y}=19.541+0.245 \mathrm{OB}^{*}$ & 0.841 & 0.784 & 0.284 \\
\hline \multicolumn{4}{|l|}{$\mathrm{OB}$} \\
\hline$Y=48.245-0.289$ Residual $*$ & 0.982 & 0.812 & 0.198 \\
\hline \multicolumn{4}{|l|}{$\mathrm{MnOx}$} \\
\hline $\mathrm{Y}=123.497+4.610$ Am_FeOx* & 0.901 & 0.851 & 0.932 \\
\hline \multicolumn{4}{|l|}{ Am_FeOx } \\
\hline $\mathrm{Y}=124.56+0.299 \mathrm{MnOx} *$ & 0.891 & 0.825 & 0.954 \\
\hline \multicolumn{4}{|l|}{ Cry_FeOx } \\
\hline $\mathrm{Y}=248.036-0.639 \mathrm{Am} \_\mathrm{FeOx} *$ & 0.903 & 0.854 & 0.923 \\
\hline \multicolumn{4}{|l|}{ Residual } \\
\hline $\mathrm{Y}=99.453-1.735 \mathrm{Am} \_\mathrm{FeOx} * *$ & 0.823 & 0.796 & 0.624 \\
\hline \multicolumn{4}{|c|}{ With physicochemical properties } \\
\hline \multicolumn{4}{|l|}{ SE } \\
\hline $\mathrm{Y}=2.318+0.024$ Clay* & 0.852 & 0.726 & 1.926 \\
\hline \multicolumn{4}{|l|}{ OB } \\
\hline $\mathrm{Y}=60.509+8.814 \mathrm{OC}^{*}$ & 0.864 & 0.746 & 4.110 \\
\hline \multicolumn{4}{|l|}{$\mathrm{MnOx}$} \\
\hline $\mathrm{Y}=180.813+29.582 \mathrm{MnO}^{* *}-5.004$ Clay* & 0.927 & 0.939 & 0.270 \\
\hline $\mathrm{Y}=0.44+34.583 \mathrm{MnO}^{* *}$ & 0.824 & 0.786 & 0.556 \\
\hline \multicolumn{4}{|l|}{$\mathrm{Am} \_\mathrm{FeOx}$} \\
\hline $\mathrm{Y}=3.355+3.45 \mathrm{Am} \_\mathrm{Fe} *-0.023$ clay $^{*}$ & 0.835 & 0.804 & 4.452 \\
\hline $\mathrm{Y}=2.094+3.910 \mathrm{Am} \_\mathrm{Fe} *$ & 0.767 & 0.589 & 7.525 \\
\hline \multicolumn{4}{|l|}{ Cry_FeOx } \\
\hline $\mathrm{Y}=39.565-4.168 \mathrm{MnO}^{*}$ & 0.777 & 0.603 & 6.385 \\
\hline \multicolumn{4}{|l|}{ Residual } \\
\hline $\mathrm{Y}=28.062-4.991 \mathrm{Am} \_\mathrm{Fe}^{*}$ & 0.896 & 0.803 & 7.781 \\
\hline
\end{tabular}

Residual Mn is mostly governed by variations in amorphous iron and oxidizable organic carbon $(90.7 \%$ in Alfisols) and amorphous iron oxide content $(80.3 \%$ in Inceptisols). Changes in residual $\mathrm{Mn}$ are mainly dictated by manganese oxide bound fraction ( $80.9 \%$ in Alfisol) and amorphous iron oxide bound fraction ( $79.6 \%$ in Inceptisols).

\section{Copper}

Total and fractions of soil copper were statistically regressed against soil physico-chemical properties. The inter relationship between $\mathrm{Cu}$ fractions have also been computed to assess the source-sink movement of soil $\mathrm{Cu}$ (Table 8-9).

The changes in soluble and exchangeable copper are mostly governed by clay content and oxidisable organic carbon of soil (88.8\% in Alfisols and $89.6 \%$ in Inceptisols) while clay content was the most important parameter in both Inceptisol (70.6\%) and Alfisol (69.4\%). Soluble and exchangeable copper was observed to be mostly impacted by changes in organically bound copper (56.8\% in Alfisols and $57 \%$ in Inceptisols)

Table 8: Relationship and Interrelationship of $\mathrm{Cu}$ fractions in Alfisol

\begin{tabular}{|c|c|c|c|}
\hline \multicolumn{2}{|c|}{$\mathbf{R}^{\mathbf{2}}$} & Adj-R $^{\mathbf{2}}$ & SEest. \\
\hline Between fractions & & & \\
\hline SE & 0.733 & 0.568 & 0.091 \\
\hline $\mathrm{Y}=-0.042+0.159 \mathrm{OB}^{*}$ & & & \\
\hline OB & 0.924 & 0.907 & 0.047 \\
\hline $\mathrm{Y}=26.035-1.016$ Residual $^{* *}-6.733 \mathrm{MnOx}^{*}$ & 0.884 & 0.782 & 0.119 \\
\hline $\mathrm{Y}=25.568-1.274$ Residual & \\
\hline MnOx & & & \\
\hline
\end{tabular}




\begin{tabular}{|c|c|c|c|}
\hline $\mathrm{Y}=0.01+0.089$ Am_FeOx** & 0.986 & 0.965 & 0.124 \\
\hline \multicolumn{4}{|l|}{$\mathrm{Am} \_\mathrm{FeOx}$} \\
\hline $\mathrm{Y}=-0.018+13.768 \mathrm{MnOx}^{*}$ & 0.935 & 0.916 & 0.049 \\
\hline \multicolumn{4}{|l|}{ Cry_FeOx } \\
\hline $\mathrm{Y}=10.580-0.692 \mathrm{Am} \_\mathrm{FeOx} *$ & 0.973 & 0.949 & 0.003 \\
\hline \multicolumn{4}{|l|}{ Residual } \\
\hline $\mathrm{Y}=25.024-0.95 \mathrm{MnOx}^{* *}-6.125 \mathrm{Am}_{-} \mathrm{FeOx} *$ & 0.988 & 0.976 & 0.046 \\
\hline $\mathrm{Y}=19.337-0.707 \mathrm{MnOx}^{* *}$ & 0.949 & 0.901 & 0.089 \\
\hline \multicolumn{4}{|c|}{ With physicochemical properties } \\
\hline \multicolumn{4}{|l|}{ SE } \\
\hline $\mathrm{Y}=-0.278+0.024$ Clay* $+0.0119 \mathrm{OC}^{*}$ & 0.926 & 0.888 & 0.052 \\
\hline $\mathrm{Y}=0.1+0.038$ Clay $* *$ & 0.716 & 0.694 & 0.087 \\
\hline \multicolumn{4}{|l|}{$\mathrm{OB}$} \\
\hline $\mathrm{Y}=-1.462+3.899 \mathrm{OC}^{* *}-0.489$ Am_Fe* & 0.938 & 0.920 & 0.468 \\
\hline $\mathrm{Y}=2.104+3.899 \mathrm{OC}^{* *}$ & 0.780 & 0.752 & 0.823 \\
\hline \multicolumn{4}{|l|}{$\mathrm{MnOx}$} \\
\hline $\mathrm{Y}=0.546+0.089 \mathrm{MnO}^{*}$ & 0.652 & 0.589 & 0.091 \\
\hline \multicolumn{4}{|l|}{ Am_FeOx } \\
\hline $\mathrm{Y}=-0.315+0.681 \mathrm{Am} \_\mathrm{Fe}^{* *}-1.514 \mathrm{MnO}^{*}$ & 0.958 & 0.946 & 0.303 \\
\hline $\mathrm{Y}=0.047+0.651$ Am_Fe* & 0.756 & 0.726 & 0.681 \\
\hline \multicolumn{4}{|l|}{ Cry_FeOx } \\
\hline $\mathrm{Y}=6.408-0.448 \mathrm{Am} \_\mathrm{Fe}^{*}$ & 0.940 & 0.923 & 0.312 \\
\hline \multicolumn{4}{|l|}{ Residual } \\
\hline $\mathrm{Y}=8.917-1.992 \mathrm{MnO}^{*}$ & 0.778 & 0.750 & 0.775 \\
\hline
\end{tabular}

Organically bound copper (OB-Cu) content is mostly governed by oxidizable organic carbon and amorphous iron oxide content in Alfisols (92\%) while in Inceptisols, oxidizable organic carbon largely controls the variation of OB-Cu (78.7\%) Among Cu fractions, residual and manganese oxide-bound copper were the major contributories (dictates 90.7\% variation of OB-Cu in Alfisol and $91.7 \%$ in Inceptisol). Variations in $\mathrm{MnOx}-\mathrm{Cu}$ are mostly governed by manganese oxide content of soil (58.9\% in Alfisols and $65.5 \%$ in Inceptisols) and amorphous iron oxide bound $\mathrm{Cu}$ fraction (96.5\% in Alfisols and $95.3 \%$ in Inceptisols). Amorphous iron oxide bound fraction is largely impacted by changes in amorphous iron oxide and manganese oxide content of Alfisols (94.6\%) and amorphous iron oxide content and oxidizable organic content of Inceptisols (83.3\%). Manganese oxide bound $\mathrm{Cu}$ fractions was observed to dictate variations in amorphous iron oxide bound $\mathrm{Cu}(91.6 \%$ in Alfisols and $91.3 \%$ in Inceptisols).

Crystalline iron oxide bound fraction is mostly affected by variations in amorphous iron oxide content of Alfisols $(92.3 \%)$ and by variations in amorphous iron oxide and oxidisable organic carbon of Inceptisols $(97.5 \%)$. Cry_FeOx$\mathrm{Cu}$ was observed further to be largely governed by changes in amorphous iron oxide bound fraction $(94.9 \%$ in Alfisols and $89.4 \%$ in Inceptisols). Residual $\mathrm{Cu}$ is mostly governed by variations in manganese oxide (75\% in Alfisols and $80.3 \%$ in Inceptisols). Soil organic carbon, further, emerged as another important contributor to changes in residual $\mathrm{Cu}$ in Inceptisols. Changes in residual $\mathrm{Cu}$ is mainly dictated by amorphous iron oxide bound fraction $(97.6 \%$ in Alfisols and $92.5 \%$ in Inceptisols).

Table 9: Relationship and Interrelationship of $\mathrm{Cu}$ fractions in Inceptisols

\begin{tabular}{|c|c|c|c|}
\hline & $\mathbf{R}^{2}$ & Adj-R ${ }^{2}$ & SEest. \\
\hline \multicolumn{4}{|l|}{ Between fractions } \\
\hline \multicolumn{4}{|l|}{ SE } \\
\hline $\mathrm{Y}=-0.049+0.172 \mathrm{OB}^{*}$ & 0.738 & 0.570 & 0.012 \\
\hline \multicolumn{4}{|l|}{$\mathrm{OB}$} \\
\hline $\mathrm{Y}=32.035-2.019$ Residual ${ }^{* *}-8.635 \mathrm{MnOx} *$ & 0.934 & 0.917 & 0.048 \\
\hline $\mathrm{Y}=29.547-1.547$ Residual $^{* *}$ & 0.881 & 0.795 & 0.169 \\
\hline \multicolumn{4}{|l|}{$\mathrm{MnOx}$} \\
\hline $\mathrm{Y}=0.01+0.079 \mathrm{Am} \_\mathrm{FeOx} * *$ & 0.986 & 0.953 & 0.006 \\
\hline \multicolumn{4}{|l|}{ Am_FeOx } \\
\hline $\mathrm{Y}=-0.018+13.968 \mathrm{MnOx}^{*}$ & 0.935 & 0.913 & 0.049 \\
\hline \multicolumn{4}{|l|}{ Cry_FeOx } \\
\hline $\mathrm{Y}=10.580-0.492 \mathrm{Am} \_\mathrm{FeOx} *$ & 0.973 & 0.949 & 0.003 \\
\hline \multicolumn{4}{|l|}{ Residual } \\
\hline $\mathrm{Y}=25.024-0.95 \mathrm{Am} \_\mathrm{FeOx} * *-6.125 \mathrm{MnOx} *$ & 0.988 & 0.925 & 0.046 \\
\hline $\mathrm{Y}=19.337-0.707$ Am_FeOx* & 0.949 & 0.901 & 0.089 \\
\hline \multicolumn{4}{|c|}{ With physicochemical properties } \\
\hline \multicolumn{4}{|l|}{ SE } \\
\hline $\mathrm{Y}=-0.292+0.014$ Clay $* *+0.251 \mathrm{OC}^{*}$ & 0.917 & 0.896 & 0.043 \\
\hline $\mathrm{Y}=0.018+0.001$ Clay $* *$ & 0.738 & 0.706 & 0.113 \\
\hline \multicolumn{4}{|l|}{$\mathrm{OB}$} \\
\hline $\mathrm{Y}=0.66+0.968 \mathrm{OC}^{*}$ & 0.811 & 0.787 & 1.208 \\
\hline \multicolumn{4}{|l|}{$\mathrm{MnOx}$} \\
\hline $\mathrm{Y}=0.54+0.247 \mathrm{MnO}^{*}$ & 0.670 & 0.655 & 0.026 \\
\hline
\end{tabular}




\begin{tabular}{|c|c|c|c|}
\hline Am_FeOx & & & \\
\hline $\mathrm{Y}=0.082+4.705 \mathrm{Am} \_F \mathrm{Fe}^{*}-0.369 \mathrm{OC}^{*}$ & 0.87 & 0.833 & 0.483 \\
\hline $\mathrm{Y}=2.497+4.152 \mathrm{Am} \_\mathrm{Fe}$ & 0.718 & 0.683 & 0.666 \\
\hline Cry_FeOx & & & \\
\hline $\mathrm{Y}=8.184-1.686 \mathrm{Am} \_F \mathrm{Fe}^{* *}-0.02 \mathrm{OC}^{*}$ & 0.981 & 0.975 & 0.234 \\
\hline $\mathrm{Y}=10.571-5.682 \mathrm{Am} \_\mathrm{Fe}$ & 0.795 & 0.770 & 0.712 \\
\hline Residual & & & \\
\hline $\mathrm{Y}=9.038-4.193 \mathrm{MnO} *-1.536 \mathrm{OC}^{*}$ & 0.902 & 0.890 & 1.384 \\
\hline $\mathrm{Y}=14.268-5.478 \mathrm{MnO} *$ & 0.845 & 0.803 & 2.365 \\
\hline
\end{tabular}

\section{Iron}

Total and fractions of soil iron were statistically regressed against soil physico-chemical properties. The inter relationship between Fe fractions have also been computed to assess the source-sink movement of soil Fe (Table 10-11).

The changes in soluble and exchangeable iron (SE-Fe) are mostly governed by clay content and soil $\mathrm{pH}$ in Alfisols $(86.7 \%)$ and Inceptisols $(97.1 \%)$ while clay content was the most important parameter in Inceptisol (81.4\%) and Alfisol $(68.4 \%)$. Soluble and exchangeable iron was observed to be mostly impacted by changes in organically bound iron $(46.6 \%$ in Alfisols and $47.4 \%$ in Inceptisols).

The changes in soluble and exchangeable iron (SE-Fe) are mostly governed by clay content and soil $\mathrm{pH}$ in Alfisols $(86.7 \%)$ and Inceptisols $(97.1 \%)$ while clay content was the most important parameter in Inceptisol (81.4\%) and Alfisol $(68.4 \%)$. Soluble and exchangeable iron was observed to be mostly impacted by changes in organically bound iron $(46.6 \%$ in Alfisols and $47.4 \%$ in Inceptisols).

Table 10: Relationship and Interrelationship of Fe fractions in Alfisols

\begin{tabular}{|c|c|c|c|}
\hline & $\mathbf{R}^{2}$ & Adj- $\mathbf{R}^{2}$ & SEest. \\
\hline \multicolumn{4}{|l|}{ Between fractions } \\
\hline \multicolumn{4}{|l|}{ SE } \\
\hline $\mathrm{Y}=31.667+0.260 \mathrm{OB}^{*}$ & 0.683 & 0.466 & 116.67 \\
\hline \multicolumn{4}{|l|}{ OB } \\
\hline $\mathrm{Y}=408.751-0.363 \mathrm{MnOx}^{* *}$ & 0.831 & 0.690 & 233.16 \\
\hline \multicolumn{4}{|l|}{$\mathrm{MnOx}$} \\
\hline $\mathrm{Y}=-536.632+0.975$ Cry_FeOx* & 0.956 & 0.914 & 281.97 \\
\hline \multicolumn{4}{|l|}{ Am_FeOx } \\
\hline$Y=-193.571+0.425$ Residual $*$ & 0.961 & 0.921 & 320.54 \\
\hline \multicolumn{4}{|l|}{ Cry_FeOx } \\
\hline $\mathrm{Y}=536.098+0.717$ Am_FeOx $* *-0.218$ Residual $*$ & 0.977 & 0.955 & 213.45 \\
\hline $\mathrm{Y}=831.711+0.938 \mathrm{Am} \_\mathrm{FeOx} * *$ & 0.956 & 0.914 & 276.54 \\
\hline \multicolumn{4}{|l|}{ Residual } \\
\hline $\mathrm{Y}=225.024-1.111 \mathrm{Am} \_\mathrm{FeOx} *$ & 0.925 & 0.856 & 496.54 \\
\hline \multicolumn{4}{|l|}{ With physicochemical properties } \\
\hline \multicolumn{4}{|l|}{ SE } \\
\hline $\mathrm{Y}=48.316-3.654$ Clay** $-2.124 \mathrm{pH}^{*}$ & 0.909 & 0.867 & 82.64 \\
\hline $\mathrm{Y}=124.318+5.129$ Clay $* *$ & 0.756 & 0.684 & 147.34 \\
\hline \multicolumn{4}{|l|}{$\mathrm{OB}$} \\
\hline $\mathrm{Y}=-663.579+172.555 \mathrm{OC}^{*}-46.32 \mathrm{MnO}^{*}$ & 0.918 & 0.846 & 177.91 \\
\hline $\mathrm{Y}=466.8+124.750 \mathrm{OC}^{*}$ & 0.745 & 0.585 & 280.35 \\
\hline \multicolumn{4}{|l|}{$\mathrm{MnOx}$} \\
\hline $\mathrm{Y}=1.893+282.110 \mathrm{MnO} *-5.5 \mathrm{Am} \_\mathrm{Fe}^{*}$ & 0.879 & 0.858 & 209.45 \\
\hline $\mathrm{Y}=232.034+64.656 \mathrm{MnO} * *$ & 0.852 & 0.805 & 295.30 \\
\hline \multicolumn{4}{|l|}{ Am_FeOx } \\
\hline $\mathrm{Y}=-3246.937+535.311$ Am_Fe* & 0.854 & 0.814 & 349.15 \\
\hline \multicolumn{4}{|l|}{ Cry_FeOx } \\
\hline $\mathrm{Y}=611.760-72.206 \mathrm{Am} \_\mathrm{Fe} *$ & 0.769 & 0.739 & 232.37 \\
\hline \multicolumn{4}{|l|}{ Residual } \\
\hline $\mathrm{Y}=3192.510-84.773 \mathrm{Am} \_\mathrm{Fe} *-61.047 \mathrm{OC}^{*}$ & 0.886 & 0.872 & 275.39 \\
\hline $\mathrm{Y}=2779.229-74.773$ Am_Fe * & 0.826 & 0.682 & 737.79 \\
\hline
\end{tabular}

Organically bound iron (OB-Fe) content is mostly governed by oxidizable organic carbon and manganese oxide content in Alfisols (84.6\%) while in Inceptisols, oxidizable organic carbon largely controls the variation of OB-Fe $(84.3 \%)$. Among Fe fractions, manganese oxide-bound iron was the major contributory (dictating 69\% variation of $\mathrm{OB}-\mathrm{Fe}$ in Alfisol and $63.7 \%$ in Inceptisol. Variations in MnOx-Fe are mostly governed by manganese oxide content and amorphous iron oxide content of soil (85.8\% in Alfisols) and manganese oxide content ( $75.4 \%$ in Inceptisols) and crystalline iron oxide bound Fe fraction was major contributor (91.4\% in Alfisols and $92.1 \%$ in Inceptisols).
Amorphous iron oxide bound fraction is largely impacted by changes in amorphous iron oxide content $(81.4 \%$ in Alfisol and $72.4 \%$ in Inceptisol). Residual iron fractions was observed to mostly dictate variations in amorphous iron oxide bound $\mathrm{Fe}(92.1 \%$ in Alfisols and $82.1 \%$ in Inceptisols). Crystalline iron oxide bound fraction is mostly affected by variations in amorphous iron oxide content of Alfisols $(73.9 \%)$ and by variations in amorphous iron oxide and manganese oxide content of Inceptisols (89.6\%) while amorphous iron oxide was the major contributory (74.4\%). Cry_FeOx-Fe was observed further to be largely governed by changes in amorphous iron oxide bound and residual fractions 
(95.5\% in Alfisols) of which amorphous iron oxide bound fraction is the dominant $(91.4 \%)$ contributor.

Table 11: Relationship and Interrelationship of Fe fractions in Inceptisols

\begin{tabular}{|c|c|c|c|}
\hline & $\mathbf{R}^{2}$ & Adj-R $\mathbf{R}^{2}$ & SEest. \\
\hline \multicolumn{4}{|c|}{ Between fractions } \\
\hline \multicolumn{4}{|l|}{ SE } \\
\hline $\mathrm{Y}=33.687+0.390 \mathrm{OB}^{*}$ & 0.693 & 0.474 & 102.68 \\
\hline \multicolumn{4}{|l|}{$\mathrm{OB}$} \\
\hline $\mathrm{Y}=440.859-0.398 \mathrm{MnOx}^{* *}$ & 0.831 & 0.637 & 233.16 \\
\hline \multicolumn{4}{|l|}{$\mathrm{MnOx}$} \\
\hline $\mathrm{Y}=-456.984+1.234$ Cry_FeOx* & 0.934 & 0.921 & 319.87 \\
\hline \multicolumn{4}{|l|}{ Am_FeOx } \\
\hline$Y=-200.98+0.697$ Residual $*$ & 0.924 & 0.897 & 345.26 \\
\hline \multicolumn{4}{|l|}{ Cry_FeOx } \\
\hline $\mathrm{Y}=852.711-1.254$ Am_FeOx $* *$ & 0.889 & 0.821 & 384.35 \\
\hline \multicolumn{4}{|l|}{ Residual } \\
\hline $\mathrm{Y}=309.024-2.956$ Am_FeOx* & 0.934 & 0.874 & 423.56 \\
\hline \multicolumn{4}{|c|}{ With physicochemical properties } \\
\hline \multicolumn{4}{|l|}{ SE } \\
\hline $\mathrm{Y}=673.731+4.624$ Clay**- $4.710 \mathrm{pH}^{* *}$ & 0.985 & 0.971 & 69.23 \\
\hline $\mathrm{Y}=42.316+12.634$ Clay $* *$ & 0.902 & 0.814 & 160.81 \\
\hline \multicolumn{4}{|l|}{$\mathrm{OB}$} \\
\hline $\mathrm{Y}=-1846.497+424.326 \mathrm{OC}^{* *}$ & 0.918 & 0.843 & 479.64 \\
\hline \multicolumn{4}{|l|}{$\mathrm{MnOx}$} \\
\hline $\mathrm{Y}=-690.934+1911.336 \mathrm{MnO}^{*}$ & 0.868 & 0.754 & 551.78 \\
\hline \multicolumn{4}{|l|}{ Am_FeOx } \\
\hline $\mathrm{Y}=-859.621+487.254 \mathrm{Am}_{-} \mathrm{Fe}^{* *}$ & 0.761 & 0.724 & 428.08 \\
\hline \multicolumn{4}{|l|}{ Cry_FeOx } \\
\hline $\mathrm{Y}=1000.752-612.236 \mathrm{Am} \_\mathrm{Fe} *-551.825 \mathrm{MnO} *$ & 0.898 & 0.896 & 100.16 \\
\hline $\mathrm{Y}=2867.75-428.757$ Am_Fe* & 0.819 & 0.744 & 488.85 \\
\hline \multicolumn{4}{|l|}{ Residual } \\
\hline $\mathrm{Y}=6037.938-74.194 \mathrm{Am} \_\mathrm{Fe}^{* *}$ & 0.867 & 0.835 & 671.03 \\
\hline
\end{tabular}

In Inceptisol, amorphous iron oxide bound fraction was the sole contributor $(82.1 \%)$. Residual Fe is mostly governed by variations in amorphous iron content and oxidizable organic carbon content in Alfisols (87.2\%) of which amorphous iron is the major contributory $(68.2 \%)$ while in Inceptisols, amorphous iron content was the sole determinant $(83.5 \%)$. Change in residual $\mathrm{Cu}$ is mainly dictated by amorphous iron oxide bound fraction $(85.6 \%$ in Alfisols and $87.4 \%$ in Inceptisols).

\section{Zinc}

Total and fractions of soil zinc were statistically regressed against soil physico-chemical properties. The inter relationship between $\mathrm{Zn}$ fractions have also been computed to assess the source-sink movement of soil $\mathrm{Zn}$ (Table 12-13).

The changes in soluble and exchangeable zinc are mostly governed by soil clay (64.9\%) in Alfisols and clay content of soil and soil $\mathrm{pH}(96.4 \%)$ in Inceptisols while clay content was the most important parameter in Inceptisol (81.3\%). Soluble and exchangeable zinc was observed to be mostly impacted by changes in organically bound zinc $(53.8 \%$ in Alfisols and
$56.7 \%$ in Inceptisols).

Organically bound zinc (OB-Zn) content is mostly governed by oxidizable organic carbon $(76.5 \%$ in Alfisols) and manganese oxide content plus oxidizable organic carbon (95.1\% in Inceptisols) and oxidizable organic carbon alone controls the variation of $\mathrm{OB}-\mathrm{Zn}(84.6 \%)$ in Inceptisols. Among Zn fractions, organically bound zinc content is mostly controlled by amorphous iron oxide bound zinc (78.2\% in Alfisols and $84.3 \%$ in Inceptisols). Variations in $\mathrm{MnOx}-\mathrm{Zn}$ are mostly governed by manganese oxide content of soil $(68.6 \%$ in Alfisols) and manganese oxide content and soil clay content (93.3\% in Inceptisol) while manganese oxide content alone controls MnOx-Zn in Inceptisol (82.6\%). MnOx-Zn was mostly governed by crystalline iron oxide bound fraction (84.7\% in Alfisol) and (86.7\% in Inceptisols). Amorphous iron oxide bound fraction is largely impacted by changes in amorphous iron oxide content in both Alfisols (91.1\%) and Inceptisols (97.7\%). Manganese oxide bound $\mathrm{Zn}$ fractions was observed to dictate variations in amorphous iron oxide bound $\mathrm{Zn}(78.2 \%$ in Alfisols and $73.2 \%$ in Inceptisols).

Table 12: Relationship and Interrelationship of $\mathrm{Zn}$ fractions in Alfisols

\begin{tabular}{|c|c|c|c|}
\hline & $\mathbf{R}^{2}$ & Adj-R ${ }^{2}$ & SEest. \\
\hline \multicolumn{4}{|c|}{ Between fractions } \\
\hline SE & & & \\
\hline$Y=-0.055+0.149 \mathrm{OB}^{*}$ & 0.733 & 0.538 & 0.141 \\
\hline \multicolumn{4}{|l|}{$\mathrm{OB}$} \\
\hline $\mathrm{Y}=0.962+0.197$ Am_FeOx $* *$ & 0.884 & 0.782 & 0.479 \\
\hline \multicolumn{4}{|l|}{$\mathrm{MnOx}$} \\
\hline $\mathrm{Y}=1.702+0.92$ Cry_FeOx $*$ & 0.92 & 0.847 & 0.108 \\
\hline Am_FeOx & & & \\
\hline $\mathrm{Y}=-0.762+3.968 \mathrm{MnOx}^{*}$ & 0.884 & 0.782 & 2.156 \\
\hline
\end{tabular}




\begin{tabular}{|c|c|c|c|}
\hline Cry_FeOx & & & \\
\hline $\mathrm{Y}=6.408-0.448 \mathrm{Am} \_\mathrm{FeOx} *$ & 0.940 & 0.923 & 0.312 \\
\hline \multicolumn{4}{|l|}{ Residual } \\
\hline $\mathrm{Y}=8.917-1.992 \mathrm{MnOx} *$ & 0.955 & 0.913 & 1.248 \\
\hline \multicolumn{4}{|c|}{ With physicochemical properties } \\
\hline \multicolumn{4}{|l|}{ SE } \\
\hline $\mathrm{Y}=0.059+0.0462$ Clay* & 0.805 & 0.649 & 0.123 \\
\hline \multicolumn{4}{|l|}{ OB } \\
\hline $\mathrm{Y}=-0.535+3.497 \mathrm{OC}^{*}$ & 0.875 & 0.765 & 0.497 \\
\hline \multicolumn{4}{|l|}{$\mathrm{MnOx}$} \\
\hline $\mathrm{Y}=0.963+8.942 \mathrm{MnO}^{*}$ & 0.828 & 0.686 & 1.551 \\
\hline \multicolumn{4}{|l|}{ Am_FeOx } \\
\hline $\mathrm{Y}=-6.828+17.130$ Am_Fe* & 0.954 & 0.911 & 1.374 \\
\hline \multicolumn{4}{|l|}{ Cry_FeOx } \\
\hline $\mathrm{Y}=19.057-1.83 \mathrm{Am} \_\mathrm{Fe}^{*}-0.001 \mathrm{EC} *$ & 0.824 & 0.795 & 1.130 \\
\hline $\mathrm{Y}=21.737-2.901 \mathrm{Am} \_\mathrm{Fe} * *$ & 0.734 & 0.754 & 1.529 \\
\hline Residual & & & \\
\hline $\mathrm{Y}=2.296-0.801 \mathrm{Am} \_\mathrm{Fe}^{*}$ & 0.768 & 0.590 & 2.706 \\
\hline
\end{tabular}

Crystalline iron oxide bound fraction is mostly affected by variations in amorphous iron oxide content and electrical conductivity in Alfisols (79.5\%) of which amorphous iron oxide content was the major contributory $(75.4 \%)$ and by variations in amorphous iron oxide alone in Inceptisols (78.2\%). Cry_FeOx-Zn was observed further to be largely governed by changes in amorphous iron oxide bound fraction (92.3\% in Alfisols and $80.3 \%$ in Inceptisols). Changes in residual $\mathrm{Zn}$ are mostly dictated by manganese oxide bound fraction in Alfisols (91.3\%) and Am_FeOx-Zn in Inceptisols $(88.7 \%)$.

Table 12: Relationship and Interrelationship of $\mathrm{Zn}$ fractions in Inceptisols

\begin{tabular}{|c|c|c|c|}
\hline & $\mathbf{R}^{2}$ & Adj-R $\mathbf{R}^{2}$ & SEest. \\
\hline \multicolumn{4}{|c|}{ Between fractions } \\
\hline \multicolumn{4}{|l|}{ 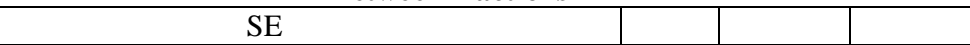 } \\
\hline$Y=-0.0635+0.193 \mathrm{OB}^{*}$ & 0.764 & 0.567 & 0.14897 \\
\hline \multicolumn{4}{|l|}{ OB } \\
\hline $\mathrm{Y}=1.262+0.254 \mathrm{Am} \_\mathrm{FeOx} * *$ & 0.861 & 0.843 & 0.59986 \\
\hline \multicolumn{4}{|l|}{$\mathrm{MnOx}$} \\
\hline $\mathrm{Y}=1.902+0.92$ Cry_FeOx $* *$ & 0.894 & 0.867 & 0.1384 \\
\hline \multicolumn{4}{|l|}{ Am_FeOx } \\
\hline $\mathrm{Y}=-0.862+5.942 \mathrm{MnOx} *$ & 0.804 & 0.732 & 2.1662 \\
\hline \multicolumn{4}{|l|}{ Cry_FeOx } \\
\hline $\mathrm{Y}=8.408-0.848 \mathrm{Am} \_\mathrm{FeOx} *$ & 0.840 & 0.803 & 0.3923 \\
\hline \multicolumn{4}{|l|}{ Residual } \\
\hline $\mathrm{Y}=11.247-2.134 \mathrm{Am} \_\mathrm{FeOx} *$ & 0.945 & 0.915 & 2.045 \\
\hline \multicolumn{4}{|c|}{ With physicochemical properties } \\
\hline \multicolumn{4}{|l|}{ SE } \\
\hline $\mathrm{Y}=0.689+0.023$ Clay $* *-0.32 \mathrm{pH}^{*}$ & 0.982 & 0.964 & 0.076 \\
\hline $\mathrm{Y}=-0.194+0.003$ Clay $* *$ & 0.902 & 0.813 & 0.164 \\
\hline \multicolumn{4}{|l|}{$\mathrm{OB}$} \\
\hline $\mathrm{Y}=-1.011+4.168 \mathrm{OC}^{* *}-0.0233 \mathrm{MnO}^{*}$ & 0.975 & 0.951 & 0.567 \\
\hline $\mathrm{Y}=3.414+3.988 \mathrm{OC}^{* *}$ & 0.92 & 0.846 & 0.938 \\
\hline \multicolumn{4}{|l|}{$\mathrm{MnOx}$} \\
\hline $\mathrm{Y}=-0.813+9.582 \mathrm{MnO}^{* *}-0.004$ Clay* & 0.987 & 0.933 & 0.270 \\
\hline $\mathrm{Y}=0.44+14.583 \mathrm{MnO}^{* *}$ & 0.884 & 0.826 & 0.556 \\
\hline \multicolumn{4}{|l|}{ Am_FeOx } \\
\hline $\mathrm{Y}=2.584+11.680 \mathrm{Am} \_\mathrm{Fe}^{*}$ & 0.989 & 0.977 & 0.822 \\
\hline \multicolumn{4}{|l|}{ Cry_FeOx } \\
\hline $\mathrm{Y}=1.018-4.821$ Am_Fe $*$ & 0.791 & 0.782 & 0.489 \\
\hline \multicolumn{4}{|l|}{ Residual } \\
\hline $\mathrm{Y}=12.783-6.019 \mathrm{Am} \_\mathrm{Fe}^{*}$ & 0.894 & 0.887 & 0.946 \\
\hline
\end{tabular}

Among all the fractions of micronutrient studied, SE fractions are reported to be most dominant one from the viewpoint of plant availability. To understand the dependency of SE fractions of all the concerned element on the soil physicochemical properties obtained from step wise regression the following scatter diagrams have been prepared. 

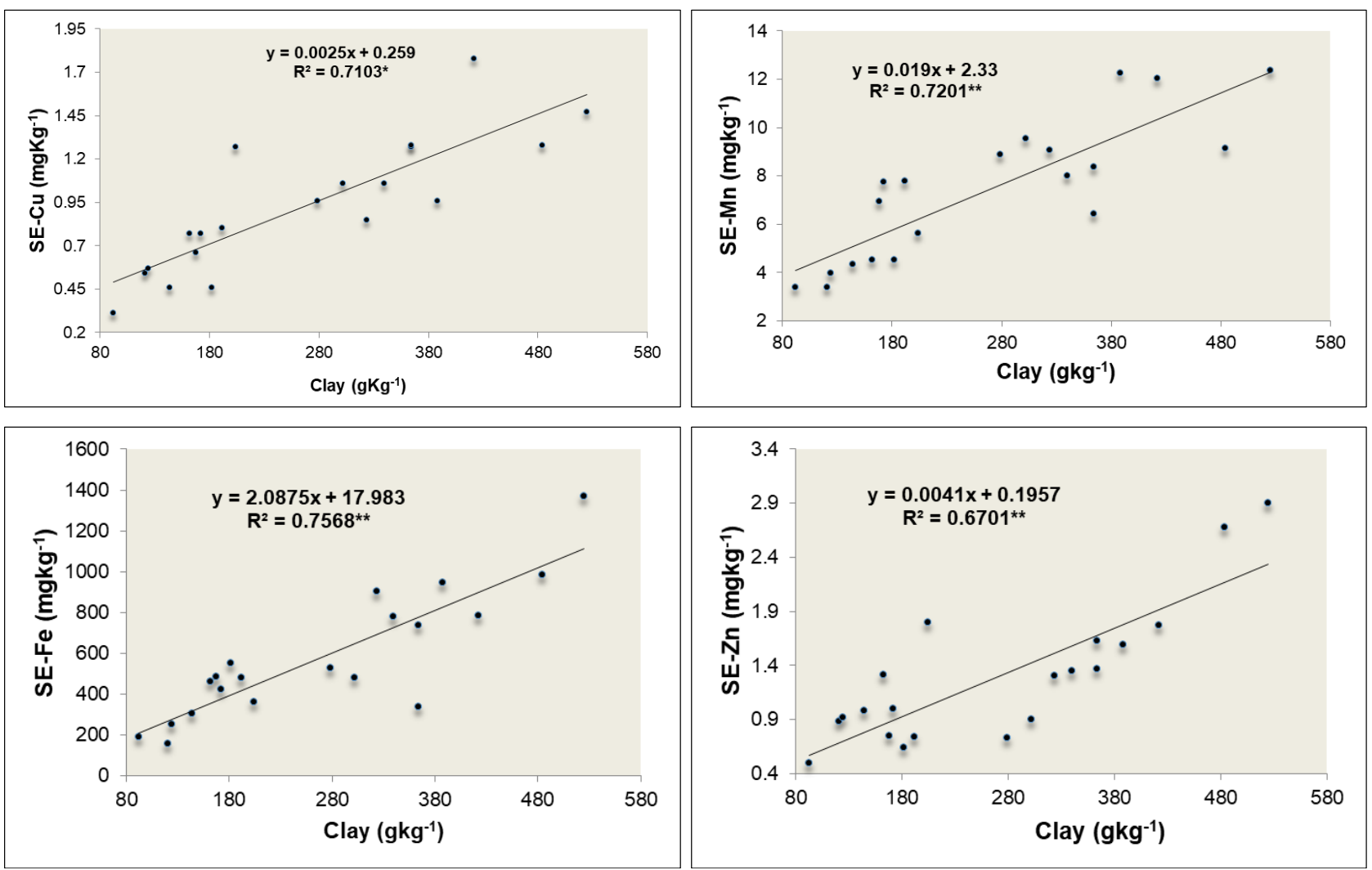

\section{Discussions}

The total manganese content of the selected soils ranged from 214.21-510.96 $\mathrm{mgkg}^{-1}$ which was more or less in good agreement with the findings of Shuman (1979) ${ }^{[57]}$ and Singh et al. (1988) ${ }^{[64]}$. The exchangeable manganese content was quite low (less than 2\%) as found by Singh et al. (1988) ${ }^{[64]}$. The manganese bound to organic matter was significantly higher than reported by Singh et al. (1988) ${ }^{[64]}$. The preference of this metal towards organic matter may be supported by their high stability constant (Gonzalez et al., 1994; Ramzan and Bhat, 2017) ${ }^{[18,55]}$. The dissolution of this iron and manganese oxide bound mineral controls mobility and bioavailability (Bhattacharya et al., 2006) ${ }^{[6]}$. The crystalline and amorphous iron bound manganese fractions was significantly lower than fractions associated with manganese oxide which possibly suggests that manganese has a tendency to be associated with Mn-oxides rather than the Fe-oxides. Such findings were also reported by Gibbs (1973) ${ }^{[66]}$, Tessier et al. (1979) ${ }^{[72]}$ and Singh et al. (1988) ${ }^{[64]}$, although opposed by Shuman (1985) [58] who found significantly higher association of manganese with organic fractions rather than Mn-oxide fractions. The residual fraction was comparatively lower than suggested by Shuman (1985) [58]; Singh et al. (1988) ${ }^{[65]}$; Kumar and Babel (2011) ${ }^{[29]}$ and Gao et al. (2018) [15].

The total copper of the selected soils were in the range 30.14$68.32 \mathrm{mg} \mathrm{kg}^{-1}$ in good agreement with the findings of

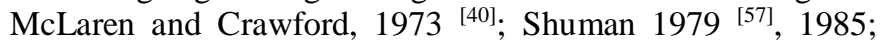
Miller et al., $1986^{[43]}$ and Singh et al., $1988^{[65]}$. The exchangeable copper content was also within the range of 1$7 \%$ as reported by Shuman, $1979^{\text {[57] }}$ and Singh et al., 1988 [65]. The low content of exchangeable copper may be attributed to leaching and plant uptake as have been reported by Filgueiras et al., $2002^{[14]}$ and Kabata-Pendias, 2011 ${ }^{[25]}$. The organically bound copper was found to be significantly higher in contrast to the findings of Singh et al., $1988^{[65]}$ but in good agreement with McLaren and Crawford, $1973^{[40]}$ and Miller and McFee, $1983^{[42]}$. This is an important consideration as has been reported by Havlin et al., $2012^{\text {[19] }}$ who observed that copper is more strongly bound to organic matter than any other micronutrient. The soil copper associated with Mn-oxide, amorphous and crystalline iron fractions were significantly higher than reported by Shuman, $1985^{[58]}$ and Singh et al., $1988^{[65]}$. Our findings can be attributed to the reports of Kabata-Pendias and Pendias, 2001 ${ }^{[26]}$ and Oviasogie et al., $2011^{[51]}$ who found that Fe-Mn bound copper was one of the major dominant fractions in alluvial soils of Nigeria. Such higher fractions of $\mathrm{Fe} / \mathrm{Mn}-\mathrm{Cu}$ has also been reported by Havlin et al., $2012{ }^{[19]}$ who suggested strong surface bonding of copper with metal oxides. However, $30-40 \%$ of the residual $\mathrm{Cu}$ fractions was telatively lower than previous findings (McLaren and Crawford, 1973; Shuman, 1979, 1985; Miller et al., 1986; Singh et al., 1988) ${ }^{[57,40,65,43,64]}$ which may be due to high weathering of copper bound minerals and transformation (Kabata-Pendias, 2011; Fathi et al., 2014; Okoli et al., 2019) $[25,12,49]$.

The total iron content of the selected soils ranged from $1.06 \%$ to $5.03 \%$ which is within the range of 0.5 to $5 \%$ normally found in soils and the exchangeable iron content was quite low (less than 2\%) as found by Singh et al., $1988^{[65]}$. The organically bound iron fraction $(8-12 \%)$ was significantly higher than obtained by Wei et al., $2010^{[75]}$ and Xing and Zhu, $2003{ }^{[76]}$ possibly having a high chemical stability constant (Mackowiak et al., 2001) ${ }^{[33]}$. The iron content in manganese oxide bound fraction was found to be low whereas the amorphous iron and crystalline iron bound fractions were found to be significantly higher than reported by others (Tessier et al., 1979; Shuman, 1985; Sims and Patrick; 1978; Singh et al., 1988; Zheng et al., 2003; Fiedler and Sommer, 2004 and Wei et al., 2010) ${ }^{[71,58,63,64,78,13,75]}$ who found that bulk of the soil iron was found in residual and crystalline iron fractions.

The total zinc content of the selected soils ranged from 30.83$108.46 \mathrm{mgkg}^{-1}$ which was found to be similar to that obtained by Singh et al., $1988^{[65]}$. In a separate study in North 
American soil, Lindsay (1972) ${ }^{[30]}$ reported a total zinc content of $10-300 \mathrm{mgkg}^{-1}$, bearing a more or less similar trend. The zinc content in the exchangeable fraction was found to be $<2 \%$ which is similar to the findings of Singh et

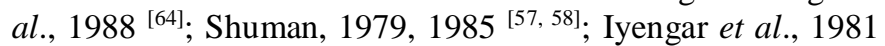
${ }^{[22]}$ and Sims, $1988^{[62]}$. The organically bound fraction of zinc (7-12\% of the total) was similar to the comparatively higher content of the said fraction in other studies (Iyengar et al., 1981; Shuman, 1985; Sims, 1988) [22, 58, 62] and in sharp contrast with findings of Singh et al., $1988{ }^{[65]}$. Zinc associated with amorphous and crystalline iron oxide fractions were higher than the manganese oxide bound fraction which may possibly indicate a greater contributory role of iron oxides in the chemistry of zinc in the soil rather than Mn-oxide fractions. Such findings were well corroborated by observations of Shuman, $1985^{[58]}$ and Singh et al., $1988^{[65]}$ but differed from Neilsen, 1990 who propounded a larger content of zinc in the Mn-oxide fraction. The undetermined pool envisaged as residual fraction was found to be in the range of $25-33 \%$ (of total $\mathrm{Zn}$ ) which was somewhat lower than reported by Iyengar et al., 1981 [22];

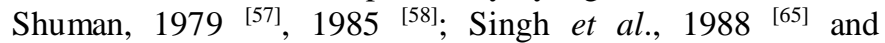
Neilsen, 1990. However, the findings that Fe-Mn bound and residual fraction of zinc attributes more than $80 \%$ of the total pool is in good agreement with the findings of Brunetto et al., $2014^{[9]}$; de Rosa Couto et al., $2014^{[10]}$ and Beygi and Jalali, $2019^{[5]}$.

The relationships and interrelationships of $\mathrm{Mn}$ fractions are quiet in good agreements with the findings of Narender et al., $2016^{[47]}$ for SE-Mn; Agbenin, $2003^{[1]}$ and Narender et al.,

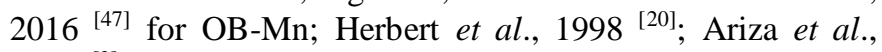
$200{ }^{[2]}$ for MnOx, Am_FeOx and Cry_FeOx fractions. The relationships and interrelationships of $\mathrm{Cu}$ fractions are found to be similar with the findings of Karin et al. 1976 and Sun et al. $2019^{[69]}$ for OB-Cu; Arora and Sekhon, 1980 ${ }^{[3]}$ for SE$\mathrm{Cu}$; McBride and Bouldin, $1984{ }^{[38]}$ for MnOx, Am_FeOx and Cry_FeOx fractions. The relationships and interrelationships of $\mathrm{Fe}$ fractions are quiet in good agreements with the findings of Shuman, $1988^{[59]}$ and Agbenin, $2003^{[1]}$ for SE-Fe; Agbenin, $2003^{[1]}$ for OB-Fe; Munch and Ottow, $1980{ }^{[46]}$ and Sun et al., $2019^{[69]}$ for $\mathrm{MnOx}$, Am_FeOx and Cry_FeOx fractions. The relationships and interrelationships of $\mathrm{Zn}$ fractions are found to be similar with the findings of LópezValdivia et al., 2002 ${ }^{[32]}$ and Soltani et al., $2015^{[67]}$ for SE-Zn; Soltani et al., $2015^{[67]}$ for OB-Zn; Katyal and Sharma, 1991

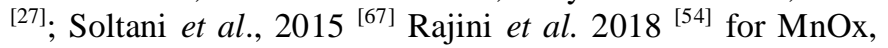
Am_FeOx and Cry_FeOx fractions.

\section{Conclusion}

The study was carried out to have a clear idea about the water-soluble and exchangeable; organically bound; manganese oxide-bound/occluded, amorphous iron oxidebound, crystalline oxide-bound fractions of the cationic micronutrients ( $\mathrm{Zn}, \mathrm{Cu}, \mathrm{Fe}, \mathrm{Mn}$ ) in the soils of two agriculturally important soil orders of West Bengal i.e. Alfisol and Inceptisol.The largest fractions of $\mathrm{Mn}, \mathrm{Cu}, \mathrm{Fe}$ and $\mathrm{Zn}$ were $\mathrm{MnOx}-\mathrm{Mn}, \mathrm{OB}-\mathrm{Cu}, \mathrm{Cry} \_\mathrm{FeO}-\mathrm{Fe}$ and Am_FeOx-Zn in both the soil orders. The soluble and exchangeable fraction which most dominantly contributes to plant availability was observed to be significantly governed by the clay content and soil $\mathrm{pH}$ and organically bound fractions.

Thus, by manipulating the concerned soil factors, the availability status of micronutrients can be altered. The agricultural significance in the form of rice cultivation can exhibit varying moisture and organic status affecting nutrient availability by changing the initial soil pools, ushering special significance to our study.

\section{References}

1. Agbenin JO. The distribution and transformation of iron and manganese in soil fractions in a savanna Alfisol under continuous cultivation. Nutrient cycling in agroecosystems. 2003; 66(3):259-270.

2. Ariza JG, Giráldez I, Sánchez-Rodas D, Morales E. Comparison of the feasibility of three extraction procedures for trace metal partitioning in sediments from south-west Spain. Science of the total environment. 2000; 246(2-3):271-283.

3. Arora CL, Sekhon GS. Influence of soil characteristics on DTPA extractable micronutrient cations in some soil series of Punjab. Journal of the Indian Society of Soil Science. 1981; 29(4):453-461.

4. ASK. Saskatchewan Agriculture. Micronutrients in Crop Production. Soils, Fertility and Nutrients. ASK Saskatchewan Agriculture, 2012.

http://www.agriculture.gov.sk.ca/

5. Beygi M, Jalali M. Assessment of trace elements $(\mathrm{Cd}$, $\mathrm{Cu}, \mathrm{Ni}, \mathrm{Zn}$ ) fractionation and bioavailability in vineyard soils from the Hamedan, Iran. Geoderma. 2019; 337:1009-1020.

6. Bhattacharya P, Chakraborty A, Chakrabarti K, Tripathy $\mathrm{S}$, Powell MA. Copper and zinc uptake by rice and accumulation in soil amended with municipal solid waste compost. Environmental Geology. 2006; 49:1064-1070.

7. Bouyoucos GJ. Hydrometer method improved for making particle size analyses of soils. Agron. J. 1962; 54(5):464465.

8. Bray RH, Kurtz LT. Determination of total, organic, and available forms of phosphorus in soils. Soil science. 1945; 59(1):39-46.

9. Brunetto G, Miotto A, Ceretta CA, Schmitt DE, Heinzen $\mathrm{J}$, de Moraes MP et al. Mobility of copper and zinc fractions in fungicide-amended vineyard sandy soils. Archives of Agronomy and Soil Science. 2014; 60(5):609-624.

10. de Rosa Couto R, Benedet L, Comin JJ, Belli Filho P, Martins SR, Gatiboni LC et al. Accumulation of copper and zinc fractions in vineyard soil in the mid-western region of Santa Catarina, Brazil. Environmental earth sciences. 2015; 73(10):6379-6386.

11. Eskandari $\mathrm{H}$. The importance of iron $(\mathrm{Fe})$ in plant products and mechanism of its uptake by plants. J Appl. Environ. Biol. Sci. 2011; 1(10):448-452.

12. Fathi H, Aryanpour H, Fathi H, Moradi H. Distribution of zinc and copper fractions in acid and alkaline (highly calcareous) soils of Iran. Sky Journal of Soil Science and Environmental Management. 2014; 3(1):6-13.

13. Fiedler S, Sommer M. Water and redox conditions in wetland soils-their influence on pedogenic oxides and morphology. Soil Science Society of America Journal. 2004; 68(1):326-335.

14. Filgueiras AV, Lavilla I, Bendicho C. Chemical sequential extraction for metal partitioning in environmental solid samples. Journal of Environmental Monitoring. 2002; 4(6):823-857.

15. Gao X, Chen S, Long A. Chemical speciation of 12 metals in surface sediments from the northern South China Sea under natural grain size, 2008.

16. Gibbs RJ. Mechanisms of trace metal transport in rivers. Science. 1973; 180(4081):71-73. 
17. Golui D, Barman M, Datta SP, Shukla AK. Fractionation of micronutrients and metals in soil. In Manual on Advance Techniques for Analysis of Nutrients and Pollutant Elements in Soil, Plant and Human (S.P. Datta, M.C. Meena, B.S. Dwivedi and A.K. Shukla, Eds). Westville Publishing House, New Delhi, 2017. (ISBN 978-93- 83491-87-2).

18. Gonzalez MJ, Ramos L, Hernandez LM. Organochlorine and heavy metal residues in the water/sediment system of the Southeast Regional Park in Madrid, Spain. International Journal of Environmental Analytical Chemistry. 1994; 57:135-140.

19. Havlin JL, Hardy DH, Gehl RJ, Spayd SE. Survey of nutrient status in Vitis vinifera grapes in North Carolina. Communications in soil science and plant analysis. 2012; 43(1-2):299-314.

20. Herbert Jr RB, Benner SG, Pratt AR, Blowes DW. Surface chemistry and morphology of poorly crystalline iron sulfides precipitated in media containing sulfatereducing bacteria. Chemical Geology. 1998; 144(1-2):8797.

21. Horváth B, Opara-Nadi O, Beese F. A Simple Method for Measuring the Carbonate Content of Soils. Soil Science Society of America Journal. 2005; 69:1066-1068. http://dx.doi.org/10.2136/sssaj2004.0010

22. Iyengar SS, Martens DC, Miller WP. Distribution and Plant Availability of Soil Zinc Fractions 1. Soil Science Society of America Journal. 1981; 45(4):735-739.

23. Jackson ML. Soil Chemical Analysis. New Delhi, Prentice Hall, 1967.

24. Jackson ML. Soil Chemical Analysis. New Delhi, Prentice Hall, 1973.

25. Kabata-Pendias A. Trace elements in soils and plants, Fourth Ed, 2011.

26. Kabata-Pendias A, Pendias H. Trace elements in soils and plants-CRC Press. Boca Raton, 2001.

27. Katyal JC, Sharma BD. DTPA-extractable and total Zn, $\mathrm{Cu}, \mathrm{Mn}$, and $\mathrm{Fe}$ in Indian soils and their association with some soil properties. Geoderma. 1991; 49:165-179.

28. Knudsen D, Peterson GA, Pratt PF. Lithium, sodium, and potassium. Methods of soil analysis. Part 2. Chemical and microbiological properties, 1982, 225-246.

29. Kumar M, Babel AL. Available micronutrient status and their relationship with soil properties of Jhunjhunu tehsil, District Jhunjhunu, Rajasthan, India. Journal of Agricultural Science (Toronto). 2011; 3(2):97-106.

30. Lindsay WL. Inorganic phase equilibria of micronutrients in soils. Micronutrients in agriculture, 1972, 41-57.

31. Lindsay WL, Norvell WA. Development of a DTPA Soil Test for Zinc, Iron, Manganese, and Copper. Soil Sci Soc Am J. 1978; 42(3):421-428.

32. Lopez-Valdivia LM, Fernandez MD, Obrador A, Alvarez JM. Zinc transformations in acidic soil and zinc efficiency on maize by adding six organic zinc complexes. Journal of agricultural and food chemistry. 2002; 50(6):1455-1460.

33. Mackowiak CL, Grossl PR, Bugbee BG. Beneficial effects of humic acid on micronutrient availability to wheat. Soil Science Society of America Journal. 2001; 65(6): 1744-1750.

34. Maleki A, Feizolahi A, Daneshian J, Naseri R, Rashnavadi R. Effect of different sources of nitrogen and zinc sulfate on grain yield and its associated traits in marigold (Calendula officinalis. L). International Journal of Biosciences. 2014; 4(6):45-52.
35. Mandal LN, Mandal B. Transformation of zinc fractions in rice soils. Soil science. 1987; 143(3):205-212.

36. Mandal LN, Mitra RR. Transformation of iron and manganese in rice soils under different moisture regimes and organic matter applications. Plant and Soil. 1982; 69(1):45-56.

37. Marschner H. Marschner's mineral nutrition of higher plants. Academic press, 2011.

38. McBride MB, Bouldin DR. Long-Term Reactions of Copper (II) in a Contaminated Calcareous Soil 1. Soil Science Society of America Journal. 1984; 48(1):56-59.

39. McKeague JA, Day J. Dithionite-and oxalate-extractable $\mathrm{Fe}$ and $\mathrm{Al}$ as aids in differentiating various classes of soils. Canadian Journal of Soil Science. 1966; 46(1):1322.

40. McLaren RG, Crawford DV. Studies on soil copper. I. The fractionation of copper in soils. J Soil Sci. 1973; 24:172-181.

41. Millaleo R, Reyes-Díaz M, Ivanov AG, Mora ML, Alberdi M. Manganese as essential and toxic element for plants: transport, accumulation and resistance mechanisms. Journal of soil science and plant nutrition. 2010; 10(4):470-481.

42. Miller WP, Mc Fee WW. Distribution of Cadmium, Zinc, Copper, and Lead in Soils of Industrial Northwestern Indiana 1. Journal of Environmental Quality. 1983; 12(1):29-33

43. Miller WP, Martens DC, Zelazny LW. Effect of Sequence in Extraction of Trace Metals from Soils 1. Soil Science Society of America Journal. 1986; 50(3):598601.

44. Moreira A, Moraes LAC, Souza LGM, Bruno IP. Bioavailability of nutrients in seeds from tropical and subtropical soybean varieties. Communications in Soil Science and Plant Analysis. 2016; 47(7):888-898.

45. Mousavi SR, Shahsavari M, Rezaei M. A general overview on manganese (Mn) importance for crops production. Australian Journal of Basic and Applied Sciences. 2011; 5(9):1799-1803.

46. Munch JC, Ottow JCG. Preferential reduction of amorphous to crystalline iron oxides by bacterial activity. Soil Science. 1980; 129(1):15-21.

47. Narender RS, Yadav K, Yadav HK. Fractionation and Distribution of Manganese in Different Cropping System and Their Relationship with Soil Properties in Haryana. Environment \& Ecology. 2016; 34(4D):25332540.

48. Nielsen JRD. Specific zinc adsorption as related to the composition and properties of clay and silt in some Danish soils. Acta Agriculturae Scandinavica. 1990; 40(1):3-9.

49. Okoli NH, Uzoho BU, Ihem EE, Okon MA. Differential speciation and availability of copper in soils derived from different parent materials in Imo State, southeastern Nigeria. Agro-Science. 2019; 18(1):7-15.

50. Olsen SR, Sommers LE. Phosphorus. AL et al. (ed.) Methods of soil analysis. Part 2. Agron. Monogr. 9. ASA and SSSA, Madison, WI, 1982, 403-430.

51. Oviasogie PO, Aghimien AE, Ndiokwere CL. Fractionation and bioaccumulation of copper and zinc in wetland soils of the Niger Delta determined by the oil palm. Chemical Speciation \& Bioavailability. 2011; 23(2):96-109. 
52. Pandey N, Pathak GC, Singh AK, Sharma CP. Enzymic changes in response to zinc nutrition. Journal of Plant Physiology. 2002; 159(10):1151-1153.

53. Phillips I, Chappie L. Assessment of a heavy metals-contaminated site using sequential extraction, TCLP, and risk assessment techniques. Soil and Sediment Contamination. 1995; 4(4):311-325.

54. Rajini SR, Narayana Rao K, Bharath Kumar KS. Distribution of zinc forms in paddy soils of upper Krishna project command area of north Karnataka. In Compendium of abstracts of the 2nd international conference on bio-resource and stress management. ANGRAU \& PJTSAU, Hyderabad, 2018, 7-10.

55. Ramzan S, Bhat MA. Distribution of Geochemical fractions of $\mathrm{Zn}, \mathrm{Fe}, \mathrm{Cu}$ and $\mathrm{Mn}$ under different landuses of Temperate Himalayas. IJCS. 2017; 5(3):734-744.

56. Shukla K, Anshumali. Soil micronutrient pools and their transfer to paddy-crops in semi-arid agro-ecosystems, Central India. Soil and Tillage Research. 2018; 180:164174.

57. Shuman LM. Zinc, manganese, and copper in soil fractions. Soil Science. 1979; 127(1):10-17.

58. Shuman LM. Fractionation method for soil microelements. Soil science. 1985; 140(1):11-22.

59. Shuman LM. Effect of organic matter on the distribution of manganese, copper, iron, and zinc in soil fractions. Soil Science. 1988; 146(3):192-198.

60. Shuman LM. Effect of organic matter on the distribution of manganese, copper, iron, and zinc in soil fractions. Soil Science. 1988; 146(3):192-198.

61. Shuman LM. Chemical forms of micronutrients in soils. Micronutrients in agriculture, (micronutrientsi2), 1991, 113-144.

62. Sims IT. Fate of heavy metals in organic wastes produced by the Delaware Solid Waste Authority. Newark Proj. Rep., Univ. of Delaware, Newark, DE, 1988.

63. Sims JL, Patrick WH. The Distribution of Micronutrient Cations in Soil under Conditions of Varying Redox Potential and $\mathrm{pH}$ 1. Soil Science Society of America Journal. 1978; 42(2):258-262.

64. Singh JP, Karwasra SPS, Singh M. Distribution and forms of copper, iron, manganese, and zinc in calcareous soils of India. Soil Science. 1988; 146(5):359-366.

65. Singh JP, Karwasra SPS, Singh M. Distribution and forms of copper, iron, manganese, and zinc in calcareous soils of India. Soil Science. 1988; 146(5):359-366.

66. Singh R, Kundu DK, Kumar A. Characterisation of Dominant Soil Subgroups of Eastern India for Formulating Water Management Strategies, 2009.

67. Soltani S, Hanafi MM, Wahid SA, Kharidah SMS. Zinc fractionation of tropical paddy soils and their relationships with selected soil properties. Chemical Speciation \& Bioavailability. 2015; 27(2):53-61.

68. Subbiah B, Asija GL. Alkaline permanganate method of available nitrogen determination. Curr Science. 1956; 25:259.

69. Sun Q, Li T, Alva AK, Li YC. Mobility and fractionation of copper in sandy soils. Environmental Pollutants and Bioavailability. 2019; 31(1):18-23.

70. Tavakoli MT, Chenari AI, Rezaie M, Tavakoli A, Shahsavari M, Mousavi SR. The importance of micronutrients in agricultural production. Advances in Environmental Biology, 2014, 31-36.
71. Tessier A, Campbell PG, Bisson M. Sequential extraction procedure for the speciation of particulate trace metals. Analytical chemistry. 1979; 51(7):844-851.

72. Tessier A, Campbell PG, Bisson M. Sequential extraction procedure for the speciation of particulate trace metals. Analytical chemistry. 1979; 51(7):844-851.

73. Viets Jr, FG. Micronutrient availability, chemistry and availability of micronutrients in soils. Journal of Agricultural and Food Chemistry. 1962; 10(3):174-178.

74. Walkley A, Black IA. An examination of the Degtjareff method for determining soil organic matter, and a proposed modification of the chromic acid titration method. Soil sci. 1934; 37(1):29-38.

75. Wei X, Shao M, Zhuang J, Horton R. Soil iron fractionation and availability at selected landscape positions in a loessial gully region of northwestern China. Soil Science \& Plant Nutrition. 2010; 56(4):617626.

www.matirkotha.net

76. Xing GX, Zhu JG. Soil Chemistry of Trace Elements and Rare Earth Elements. Science Press, Beijing (in Chinese), 2003.

77. Yadegari M, Shakerian A. Irrigation periods and Fe, Zn foliar application on agronomic characters of Boragoofficinalis, Calendula officinalis, Thymus vulgarisand Alyssum desertorum. Advances in Environmental Biology, 2014, 1054-1063.

78. Zheng Y, Zhang F, Li L. Iron availability as affected by soil moisture in intercropped peanut and maize. Journal of plant nutrition. 2003; 26(12):2425-2437. 